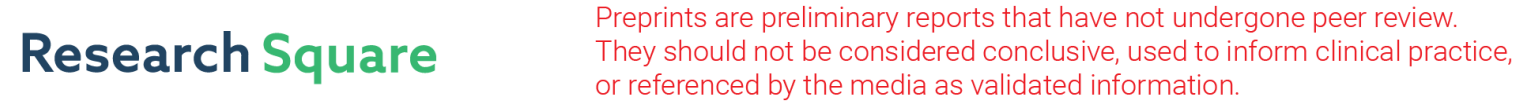 \\ Tri-valued Memristor-based Hyper-chaotic System with Hidden and Coexistent Attractors
}

\author{
Xiaoyuan Wang ( $\square$ youyuan-0213@163.com ) \\ Hangzhou Dianzi University https://orcid.org/0000-0003-0865-7827 \\ Meng Gao \\ Hangzhou Dianzi University College of Electronics and Information \\ Herbert Ho-Ching lu \\ University of Western Australia \\ Chunhua Wang \\ Hunan University
}

\section{Research Article}

Keywords: Tri-valued memristor, Hyper-chaos, Hidden attractor, Coexistent attractor, Pseudorandom number generator

Posted Date: August 5th, 2021

DOl: https://doi.org/10.21203/rs.3.rs-758374/v1

License: (c) (1) This work is licensed under a Creative Commons Attribution 4.0 International License. Read Full License 


\title{
Tri-valued Memristor-based Hyper-chaotic System with Hidden and Coexistent Attractors
}

\author{
Xiaoyuan Wang ${ }^{\bowtie}$. Meng Gao $\cdot$ Herbert Ho-Ching Iu · Chunhua Wang
}

Received: date / Accepted: date

\begin{abstract}
Recently, the nonlinear dynamics of memristor has attracted much attention. In this paper, a novel fourdimensional hyper-chaotic system (4D-HCS) is proposed by introducing a tri-valued memristor to the famous Lü system. Theoretical analysis shows that the 4D-HCS has complex chaotic dynamics such as hidden attritors and coexistent attractors, and it has larger maximum Lyapunov exponent and chaotic parameter space than the original Lü system. We also experimentally analyze the dynamics behaviors of the 4D-HCS in aspects of the phase diagram, Poincaré mapping, bifurcation diagram, Lyapunov exponential spectrum, and the correlation coefficient, and the analysis results show the complex dynamic characteristics of the proposed 4D-HCS. In addition, the comparison with binary-valued memristorbased chaotic system shows that the 4D-HCS has unique characteristics such as hyper-chaos and coexistent attractors. To show the easy implementation of the 4D-HCS, we implement the 4D-HCS in an analogue circuit-based hardware platform, and the implementation results are consistent with the theoretical analysis. Finally, using the 4D-HCS, we design a pseudorandom number generator to explore its potential application in cryptography.
\end{abstract}

Keywords Tri-valued memristor $\cdot$ Hyper-chaos $\cdot$ Hidden attractor $\cdot$ Coexistent attractor $\cdot$ Pseudorandom number generator

Xiaoyuan Wang $\bowtie$. Meng Gao

School of Electronics and Information, Hangzhou Dianzi University, Hangzhou 310018, China

E-mail: youyuan-0213@163.com

Herbert Ho-Ching Iu

School of Electrical, Electronic and Computer Engineering, The University of Western Australia, Perth 6009, Australia

Chunhua Wang

School of Computer Science and Electronic Engineering, Hunan University, Changsha 410082, China

\section{Introduction}

Chaos is a pseudorandom phenomenon produced by a certain nonlinear system. It shows many unique properties such as initial sensitivity and ergodicity. Since the first chaotic system was designed by Lorenz in 1963 [1], researchers have developed many chaotic systems and applied them to a wide body of research fields such as dynamics research [2], neural networks [3], secure communication [4-6], image encryption [7-11].

Generally, the complexity of a chaotic system is determined by its nonlinear term [12]. Memristor is a circuit element with nonlinear characteristics, and it can be used to construct chaotic circuits [13]. In 2008, Itoh and Chua first introduced the memristor into chaotic system to propose a memristive chaotic system [14]. In 2012, Wang et al. first proposed a memristor model using light dependent resistor (LDR) [15], and then designed a chaotic circuit based on the LDR memristor [16].

In recent years, chaotic systems are discovered to have many new characteristics such as hidden attractors, coexisting attractors, and multistability [17]. For example, Li et al. proposed a new memristive chaotic system with an infinite equilibrium plane in [18]. The amplitude and frequency of the system can be changed by adjusting the initial value of the internal state variable $u$ of the memristor, indicating that the chaotic system has hidden and coexisting attractors. In 2018, using the Wien-bridge chaotic circuit, Ye et al. proposed a memristive hyper-chaotic circuit with coexisting attractors [19]. In the same year, Tan et al. proposed an inductor-free memristive chaotic circuit with three line equilibria and coexisting attractors [20]. In 2019, Wang et al. proposed a chaotic oscillator using memcapacitor and meminductor [21]. The system has infinite number of equilibrium points and coexisting attractors, and is extremely sensitive to initial values. In the same year, Min et al. added an 
optimization factor $f$ to a memristive chaotic system, and then obtained a new system without equilibrium point, and realized the control of the hidden attractor [22]. In 2020, Wang et al. introduced a memristor feedback into a Lorenzlike chaotic system to obtain a hyper-chaotic system with multistability [23]. This system has rich and unique dynamic characteristics. In addition, the nonlinear and random-like behavior of chaotic systems makes them suitable for designing pseudorandom number generator (PRNG). To explore this application, Hua et al. designed a PRNG using a 2D sine chaotification system. It can generate random numbers with high randomness [24].

Up to now, most research works on memristive chaotic systems focus on binary-valued and continuous memristors. Tri-valued or multi-valued memristors can store more information and have richer characteristics than binary-valued memristors. However, tri-valued memristor and its chaotic system haven't received much attention. Therefore, it is of great significance to enrich the characteristics of chaotic systems using the tri-valued memristors. In this paper, we first introduce a tri-valued memristor model, and then propose a four-dimensional hyper-chaotic systems (4D-HCS) using the tri-valued memristor. Theoretical analysis and experimental results show the complex dynamics behaviors of the developed 4D-HCS.

This paper is organized as follows. Section 2 introduces the tri-valued memristor model and its equivalent circuit. Section 3 presents the constructed 4D-HCS, calculates its Lyapunov exponent and Lyapunov dimension, and discusses its stability and equilibrium points. Section 4 analyzes the hidden and coexistent attractors of the 4D-HCS. Section 5 compares the 4D-HCS with the binary-valued memristorbased chaotic system. Section 6 designs a hardware circuit platform for the 4D-HCS and applies it to the design of PRNG. Finally, section 7 gives the conclusions about this paper.

\section{A Tri-valued Memristor Model and Its Equivalent Circuit}

\subsection{The tri-valued memristor model}

The memristor is used to describe the relationship between the charge $q$ and the flux $\varphi$. According to the definition in [25], ideal memristors can be divided into current-controlled memristors and voltage-controlled memristors, which can be described by Eqs. (1) and (2), respectively:

$$
\left\{\begin{array}{l}
v=R(q) i \\
\frac{d q}{d t}=i
\end{array}\right.
$$

$$
\left\{\begin{array}{l}
i=G(\varphi) v \\
\frac{d \varphi}{d t}=v
\end{array}\right.
$$

where $v$ and $i$ represent the voltage across and current flowing through the memristor, $q$ and $\varphi$ mean the charge and flux on the memristor at time $t, \mathrm{R}(q)$ and $G(\varphi)$ are the memristance and memductance of the memristor.

In [26], Wang et al. proposed a voltage-controlled trivalued memristor mathematical model. It satisfies the memristor theory and is different from binary-valued and continuous memristors. The $q-\varphi$ relationship of the model is described by an asymmetric piecewise linear function, whose general expression is shown in Eq. (3):

$q=e_{0}+a_{0} \varphi+b_{0}\left|\varphi+c_{0}\right|-d_{0}\left|\varphi-c_{0}\right|$

where $a_{0}, b_{0}, c_{0}, d_{0}$ and $e_{0}$ are non-zero constant parameters and $c_{0}$ is positive. Taking the derivative of the flux $\varphi$ form Eq. (3), the $G-\varphi$ relationship of the tri-valued memristor is calculated as:

$$
\begin{aligned}
\frac{d q}{d \varphi}=G(\varphi) & =a_{0}+b_{0} \operatorname{sgn}\left(\varphi+c_{0}\right)-d_{0} \operatorname{sgn}\left(\varphi-c_{0}\right) \\
& = \begin{cases}a_{0}-b_{0}+d_{0}, & \varphi<-c_{0} \\
a_{0}+b_{0}+d_{0}, & -c_{0}<\varphi<c_{0} \\
a_{0}+b_{0}-d_{0}, & \varphi>c_{0}\end{cases}
\end{aligned}
$$

where $\operatorname{sgn}(x)$ represents the symbolic function. When $x>0$, $\operatorname{sgn}(x)=1$ and when $x<0, \operatorname{sgn}(x)=-1$.

In this paper, we set $a_{0}=2.5, b_{0}=4, c_{0}=1, d_{0}=2.5$ and $e_{0}=-$ 1.5 , according to Eqs. (3) and (4), the $\varphi$ - $q$ curve and $\varphi-G$ curve of the tri-valued memristor with three stable memductance $1 \mathrm{~S}, 9 \mathrm{~S}$ and $4 \mathrm{~S}$ can be obtained and they are shown in Fig. 1. We can see that the $\varphi-q$ curve is across the origin, and the three different slopes in the curve indicate three memductances of the memristor controlled by the voltage.

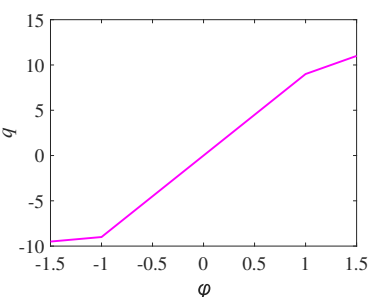

(a)

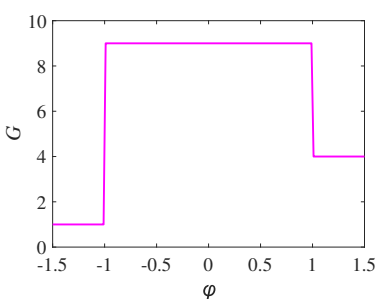

(b)
Fig. 1 Characteristic curves of voltage-controlled tri-valued memristor model: (a) $\varphi$ - $q$ curve, (b) $\varphi$ - $G$ curve

By applying a sinusoidal signal $v(t)=v_{0} \sin (2 \pi f t)$ to the above model, and taking amplitude $v_{0}=4 \mathrm{~V}$, frequency $f=$ $0.159 \mathrm{~Hz}$, initial value $\varphi(0)=-1.5$, the $v-i$ curve and timing diagram of the memristor can be obtained and shown in Fig. 2. 


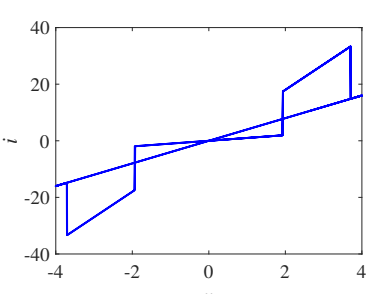

(a)

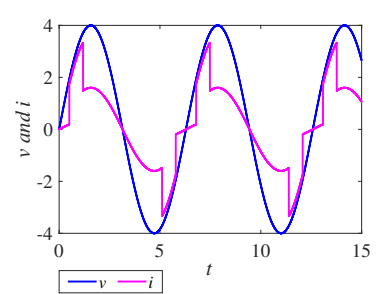

(b)
Fig. 2 The $v-i$ characteristic curve of the voltage-controlled tri-valued memristor model: (a) Pinched hysteresis loop, (b) Timing diagram

\subsection{Multiple pinch-off points analysis of the memristor}

The circuit characteristics of the memory element appear as a pinched hysteresis loop. Since the multiple pinch-off point behavior reflects the complex nonlinearity of a memristor, it is an important indicator to analyze the characteristics of memristors [27,28].

When applying a sinusoidal signal $v(t)=v_{0} \sin (\omega t)$ to the tri-valued memristor, we can obtain the flux $\varphi$ by Eq. (2) as:

$$
\begin{aligned}
\varphi(t) & =\int_{-\infty}^{t} v(\tau) d \tau=\int_{-\infty}^{t} v_{0} \sin (\omega \tau) d \tau \\
& =\varphi(0)-\frac{v_{0}}{\omega} \cos (\omega t)+\frac{v_{0}}{\omega}
\end{aligned}
$$

It can be seen from Eq. (5) that the value of the flux $\varphi$ is determined by the initial value $\varphi(0)$, input voltage amplitude $v_{0}$ and frequency $\omega$. The maximum flux $\varphi_{S}$ under the sinusoidal voltage input is obtained in half the input cycle, as shown in Eq. (6).

$\varphi_{S}=\varphi(0)+2 \frac{v_{0}}{\omega}$

For the $\varphi-q$ relationship of the piecewise linear memristor, its piecewise points $\varphi_{1}=-1$ and $\varphi_{2}=1$ show its nonlinearity, which is affected by the flux $\varphi_{S}$ and the result is expressed as the number of pinch-off points.

Let $\varphi(0)=-1.5$ and $v_{0}=4$, the memristor characteristics along the change of $\omega$ are shown as Fig. 3.

When $\omega=2, \varphi_{S}=2.5>\varphi_{2}$, and $\varphi(0)=-1.5<\varphi_{1}$, the $\varphi-q$ curve has two piecewise points $\varphi_{1}$ and $\varphi_{2}$, and the $v$ - $i$ curve has three pinch-off points. The $\varphi_{S}$ decreases with the increment of $\omega$. When $\omega=4, \varphi_{S}=0.5<\varphi_{2}$, the $\varphi$ - $q$ curve has one piecewise point $\left(\varphi_{1}\right)$, which shows a conventional single pinch-off memristor; When $\omega=20$, and $\varphi_{S}=-1.1<\varphi_{1}<\varphi_{2}$, the $\varphi-q$ curve has no piecewise point and shows a linear relationship. Then the $v-i$ relationship appears as a straight line across the origin.

It can be seen that the frequency $\omega$ affects the value of the flux $\varphi_{S}$ and further changes the nonlinear characteristics of the memristor. The experimental results show that

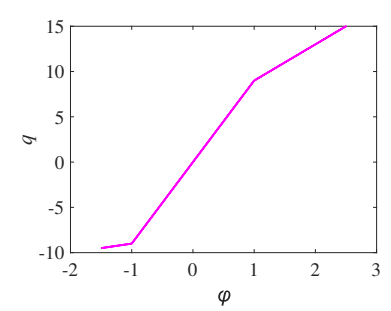

(a)

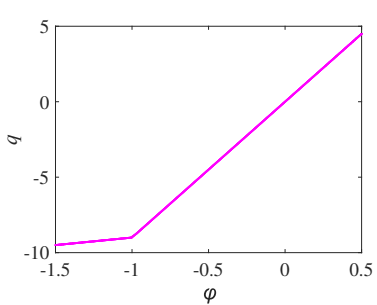

(c)

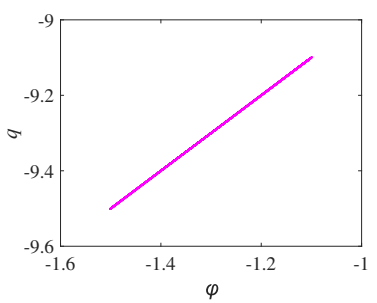

(e)

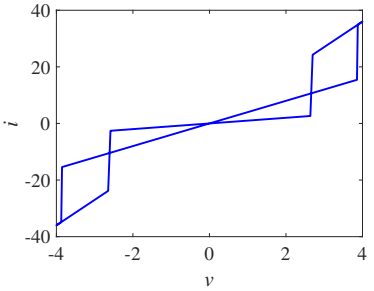

(b)

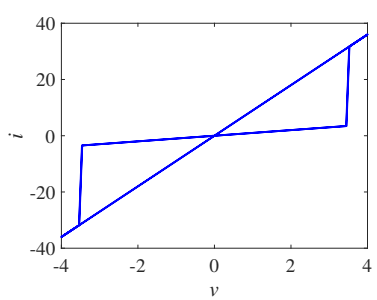

(d)

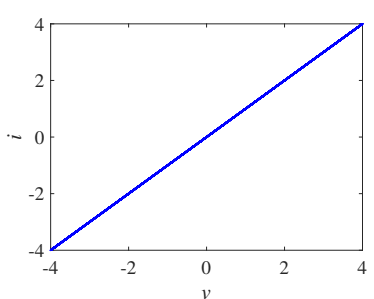

(f)
Fig. 3 The $\varphi-q$ curve and $v-i$ curve of the memristor under different $\omega$ (unit: $\mathrm{rad} / \mathrm{sec}$ ): (a) and (b) $\omega=2,(\mathbf{c})$ and (d) $\omega=4,(\mathbf{e})$ and (f) $\omega=20$

when all piecewise points $\varphi_{1}$ and $\varphi_{2}$ are included between $\varphi(0)$ and $\varphi_{S}$, the $\varphi-q$ curve has three pinch-off points and the memristor has high nonlinearity, which is a tri-valued memristor.

\subsection{Equivalent circuit of the memristor}

According to Eqs. (2)-(4), the equivalent circuit of the trivalued memristor can be designed and it is shown as Fig. 4, where the $A_{1}$ is an analogue multiplier, $U_{4}$ and $U_{5}$ are voltage comparators, and the other components are operational amplifiers.

As can be seen from Fig. $4, R_{1}, C_{1}$, and $U_{1}$ form an inverting integration circuit, which turns the input voltage $v$ into the negative flux $-\varphi$. Through the inverting addition circuit composed by $R_{2}, R_{3}, R_{4}$, and $U_{2}, \varphi+V_{1}$ can be obtained as the output of $U_{2}$. Similarly, the output at $U_{3}$ is $\varphi-V_{2}$ and the output of $U_{4}$ and $U_{5}$ are $\operatorname{sgn}(\cdot)$ in Eq. (4). Take $U_{4}$ as an example, when the input of $U_{4}$ is $\varphi+V_{1}$ and 0 , the output will be $U_{\text {sat }}$ if $\varphi+V_{1}>0$ and it will be $-U_{\text {sat }}$ if $\varphi+V_{1}<0$, where $U_{\text {sat }}$ is the saturated output voltage. For simplicity, the output of $U_{4}$ can be written as $U_{\text {sat }} \operatorname{sgn}\left(\varphi+V_{1}\right)$. Similarly, the output $U_{5}$ is $-U_{s a t} \operatorname{sgn}\left(\varphi-V_{2}\right)$. Since $R_{8}, R_{9}$, and $U_{6}$ form an invert- 

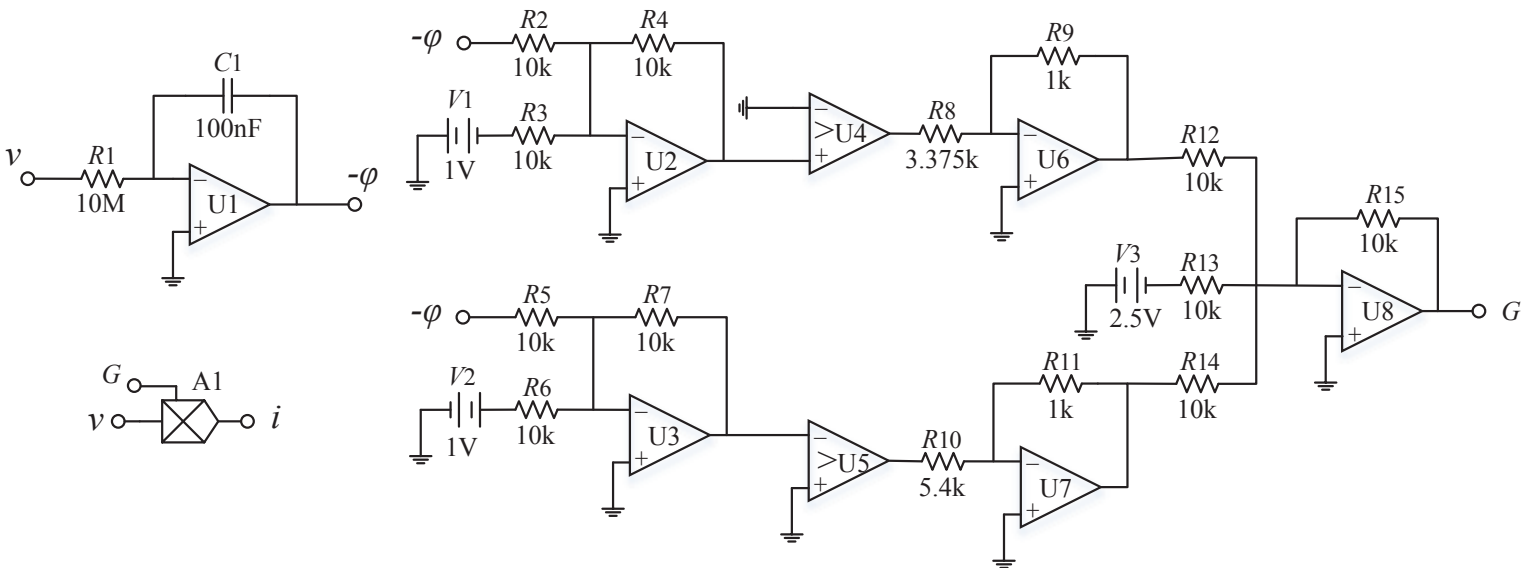

Fig. 4 Equivalent circuit of the memristor

ing proportional operational circuit, the output of $U_{6}$ is $-\left(R_{9} /\right.$ $\left.R_{8}\right) U_{\text {sat }} \operatorname{sgn}\left(\varphi+V_{1}\right)$. The output of $U_{7}$ is $\left(R_{11} / R_{10}\right) U_{\text {sat }} \operatorname{sgn}(\varphi-$ $V_{2}$ ). Since $R_{12}, R_{13}, R_{14}, R_{15}$ and $U_{8}$ form an inverting addition circuit to add the outputs of $U_{6}$ and $U_{7}$ and $-V_{3}$, the memductance $G(\varphi)$ finally is obtained from the equivalent circuit can be described as Eq. (7).

$G(\varphi)=V_{3}+\frac{R_{9}}{R_{8}} \cdot U_{s a t} \cdot \operatorname{sgn}\left(\varphi+V_{1}\right)-\frac{R_{11}}{R_{10}} \cdot U_{\text {sat }} \cdot \operatorname{sgn}\left(\varphi-V_{2}\right)$

where $U_{\text {sat }}$ is the saturated output voltage of the voltage comparators and it is set as $13.5 \mathrm{~V}$. Set $V_{1}$ and $V_{2}$ in the circuit as constant $c_{0}$ in Eq. (4), namely $1 \mathrm{~V}$ and set $V_{3}$ as constant $a_{0}$, namely $2.5 \mathrm{~V}$. The circuit parameters determined by Eq. (7) are $C_{1}=100 \mathrm{nF}, R_{1}=10 \mathrm{M} \Omega, R_{2}=R_{3}=R_{4}=R_{5}=R_{6}=R_{7}=10 \mathrm{k} \Omega$, $R_{8}=3.375 \mathrm{k} \Omega, R_{9}=R_{11}=1 \mathrm{k} \Omega, R_{10}=5.4 \mathrm{k} \Omega, R_{12}=R_{13}=R_{14}=R_{15}$ $=10 \mathrm{k} \Omega$.

After $G(\varphi)$ is obtained, the current going through the memristor can be achieved by multiply the input voltage with the $G(\varphi)$. As shown in Fig. 5, when the input voltage $v(t)=4 \sin (2 \omega f t)$ is given $(f=0.159 \mathrm{~Hz}, \varphi(0)=-1.5)$, the timing diagram of $v, i$ and $G$ are consistent with the simulation result shown in Fig. 2, which verifies the effectiveness of the equivalent circuit.

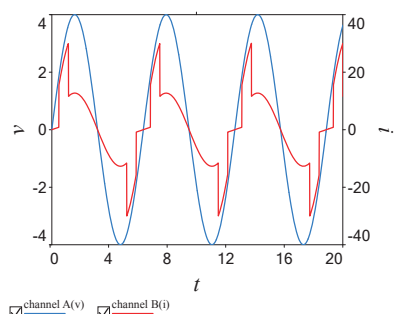

(a)

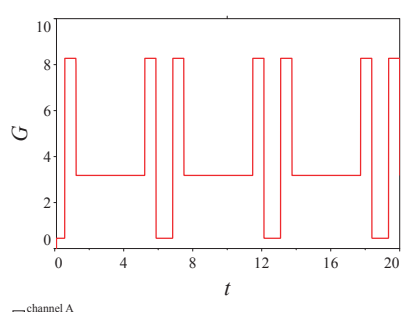

(b)
Fig. 5 Simulation results: (a) Timing diagram of $v$ and $i$; (b) Timing diagram of $G$

\section{The Four-dimensional Chaotic System}

\subsection{Construction of memristive chaotic system}

In 2002, Lü et al. proposed a new three-dimensional chaotic system called Lü system [29], which realizes a conversion between the Lorenz system [1] and Chen system [30].

As a nonlinear device, the memristors can be used to construct chaotic systems. In this paper, a new 4D hyperchaotic system (4D-HCS) is proposed by introducing the voltage-controlled tri-valued memristor model to the Lü system. The expression of the 4D-HCS is shown as follows:

$$
\left\{\begin{array}{l}
\dot{x}=a(y-x) \\
\dot{y}=c y-x z \\
\dot{z}=x y-b z-d G(w) \\
\dot{w}=z
\end{array}\right.
$$

where $x, y, z$ and $w$ are system state variables, and $a, b, c$ and $d$ are constant parameters. $G(w)$ is the memductance of the tri-valued memristor in Eq. (4). When setting these parameters as $a=40, b=5, c=24.4$ and $d=50$, and these initial values as $\left[x_{0}, y_{0}, z_{0}, w_{0}\right]=[0.01,0.01,0.01,0.01]$, the Lyapunov exponents of the 4D-HCS are calculated as $L E_{1}=4.2486$, $L E_{2}=0.0025, L E_{3}=-0.004$ and $L E_{4}=-24.8471$, and its Lyapunov dimension is calculated as $D_{L}=3.1709$ using Eq. (9). As a contrast, the Laypunov exponents of the Lü system are $L E_{1}=2.7931, L E_{2}=0.0049, L E_{3}=-23.3979$ and its Lyapunov dimension is $D_{L}=2.1196$. It is obvious that the $4 \mathrm{D}-\mathrm{HCS}$ has a higher maximum Lyapunov exponent and Lyapunov dimension than the Lü system.

$D_{L}=k+\frac{1}{\left|L E_{k+1}\right|} \sum_{i=1}^{k} L E_{i}=3+\frac{\left(L E_{1}+L E_{2}+L E_{3}\right)}{\left|L E_{4}\right|}$

The phase diagrams of the 4D-HCS under the above conditions are shown in Fig. 6, which indicates the rich dynamic behaviors of the system. 


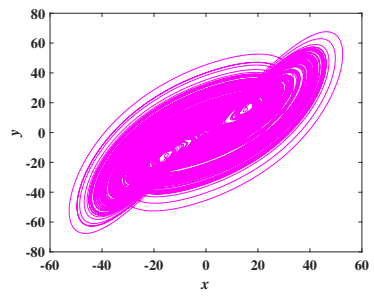

(a)

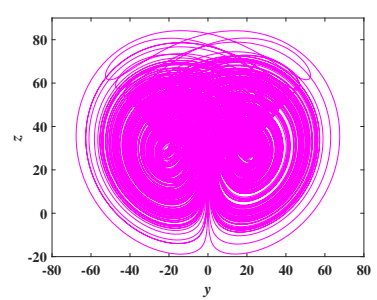

(c)

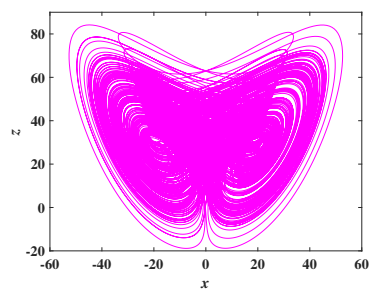

(b)

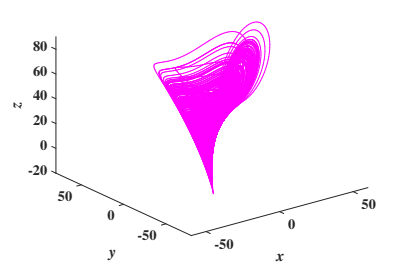

(d)
Fig. 6 Phase diagrams of the system based on the tri-valued memristor: (a) $x-y$, (b) $x-z$, (c) $y-z$, (d) $x-y-z$

\subsection{Hyper-chaotic behaiovrs}

Setting its parameters as other values, the 4D-HCS can show hyper-chaotic behaviors. Specifically, setting the system parameters as $a=12, b=-1, c=-1.2$ and $d=50$, and its initial values as $\left[x_{0}, y_{0}, z_{0}, w_{0}\right]=[0.1,0.1,0.1,0.1]$, its Lyapunov exponent are calculated as $L E_{1}=2.4566, L E_{2}=0.3086, L E_{3}=-$ $0.0064 \approx 0$ and $L E_{4}=-14.9588$, and its Lyapunov dimension $D_{L}=3.1844$. Since there are two positive Laypunov exponents, the 4D-HCS shows hyper-chaotic behaviors under this condition. The corresponding typical hyper-chaotic attractors can be obtained and shown in Fig. 7.

\subsection{Dissipative property analysis}

To study the dissipative property, we calculated the divergence of the system using Eq. (10).

$\nabla V=\frac{\partial \dot{x}}{\partial x}+\frac{\partial \dot{y}}{\partial y}+\frac{\partial \dot{z}}{\partial z}+\frac{\partial \dot{w}}{\partial w}$

when parameters $a=12, b=-1, c=-1.2$ and $d=50$, the $\nabla V=-a$ $+c-b=-12.2<0$. Then the system is dissipative and satisfies the conditions for generating chaotic attractors.

\subsection{Equilibrium point and stability analysis}

To analyze the stability of the 4D-HCS, the equilibrium point of the system must be calculated. Let the right part of Eq. (8) equal to zero, and the system parameters $a, b, c$ and $d$ are non-zero values, we can obtain an equilibrium point $O=\{x=y=z=0, G(w)=0\}$. However, the tri-valued memristor model defined by Eqs. (3) and (4) shows that the memductance $G(w) \neq 0$, which indicates that the 4D-HCS based on the tri-valued memristor has no equilibrium point. Thus, the chaotic attractors generated by the 4D-HCS are all hidden attractors.

\subsection{Timing diagram and Poincaré mapping analyses}

The Timing diagram of the 4D-HCS is shown in Fig. (8), which indicates that the 4D-HCS shows pseudorandom and aperiodic behaviors. Fig. 9(a) and Fig. 9(b) show the Poincaré mapping on the $x-z$ plane with $y=-10$ and the $x-y$ plane with $z=10$, respectively. As can be seen, the Poincaré mapping are composed of dense points with hierarchical structures.

\section{Dynamical Property Analysis}

4.1 Influence of system parameters on dynamic characteristics

The behaviors of a chaotic system are extremely sensitive to the change of its parameters. Therefore, it is necessary to study the influence of parameter variation on system dynamics.

\subsubsection{Influence of the parameter a}

For the 4D-HCS, we first study its behaviors when its parameter $a$ increases within $[1,12]$ by fixing the initial values $\left[x_{0}, y_{0}, z_{0}, w_{0}\right]=[0.1,0.1,0.1,0.1]$, and its other parameters $b=-1, c=-1.2$ and $d=50$. The corresponding Lyapunov exponent spectrum and bifurcation diagram can be obtained and shown in Fig. 10 and Fig. 11, respectively.

It can be seen from Fig. 10 that with the increment of parameter $a$, the 4D-HCS has different Lyapunov exponents, and thus it exhbits different states. The bifurcation diagram in Fig. 11 shows that the 4D-HCS transforms from the periodic state first to the chaotic state, and then to the hyperchaotic state, as the parameter $a$ increases. The specific evolution process is shown in Table 1 , and the representative phase diagrams in $x-y$ plane are shown in Fig. 12.

Table 1 Different system states corresponding to different values of $a$

\begin{tabular}{cccccl}
\hline Value of $a$ & $L E_{1}$ & $L E_{2}$ & $L E_{3}$ & $L E_{4}$ & State \\
\hline 1.4 & 0.0009 & -0.0029 & -0.7991 & -0.7989 & Period \\
2.1 & 0.5289 & -0.0013 & -0.0056 & -2.8221 & $\begin{array}{l}\text { Chaotic } \\
8\end{array}$ \\
\hline
\end{tabular}




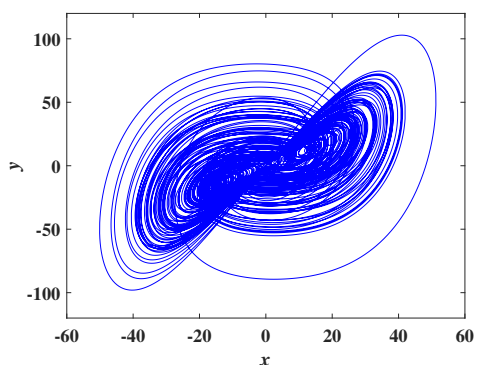

(a)

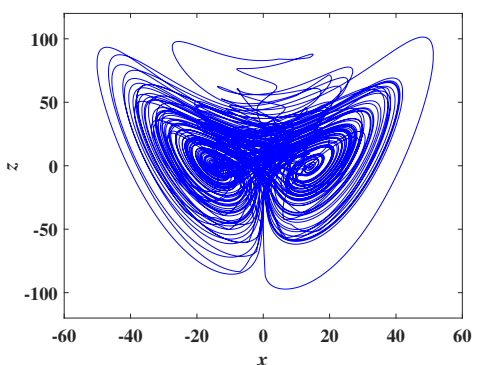

(b)

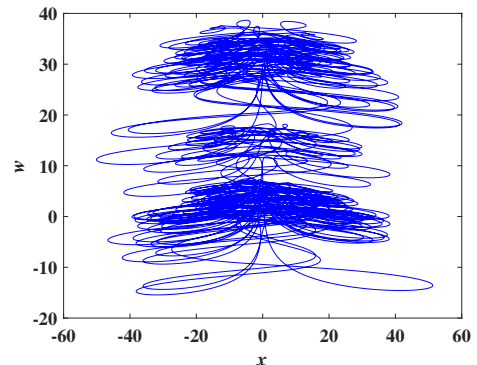

(c)

Fig. 7 Phase diagram of the hyper-chaotic attractors: (a) $x-y$, (b) $x-z$, (c) $x-w$
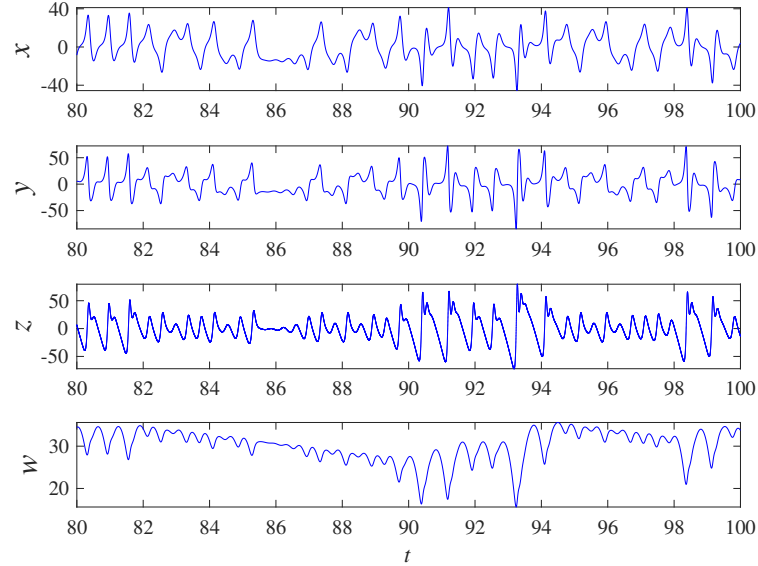

Fig. 8 Timing diagram of $x, y, z$ and $w$

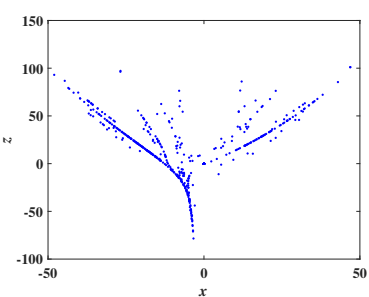

(a)

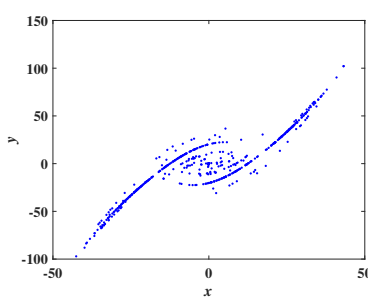

(b)
Fig. 9 Poincaré mapping: (a) $y=-10$, (b) $z=10$

\subsubsection{Influence of the parameter $c$}

Similar to the analysis of parameter $a$, we fix the initial values $\left[x_{0}, y_{0}, z_{0}, w_{0}\right]=[0.1,0.1,0.1,0.1]$, and parameters $a=12$, $b=-1$ and $d=50$ in the 4D-hCS and calculate its Lyapunov exponent spectrum and bifurcation diagram with the change of parameter $c$. Fig. 13 and Fig. 14 show the calculation results. As can be seen from Fig. 13, the Lyapunov exponent changes with the variation of the parameter $c$, and the system switches between the chaos and hyper-chaotic when $c$

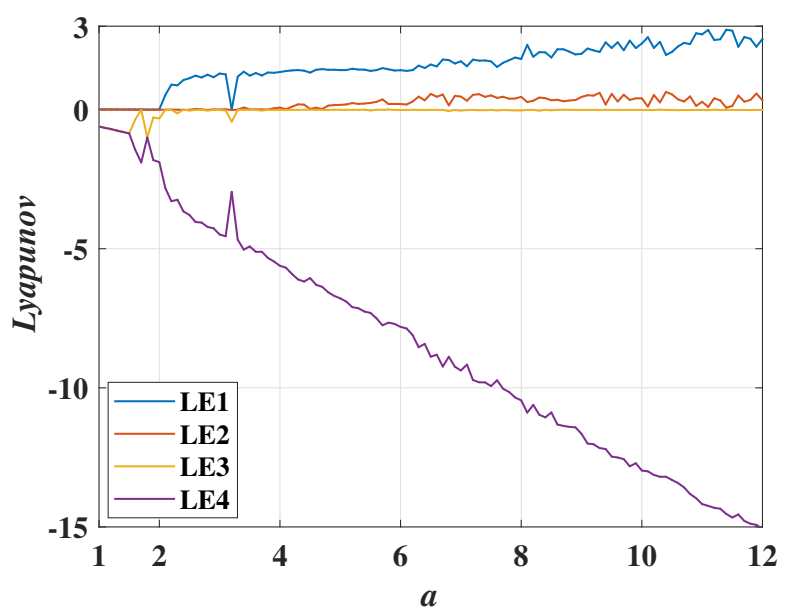

Fig. 10 Lyapunov exponent spectrum corresponding to $a$

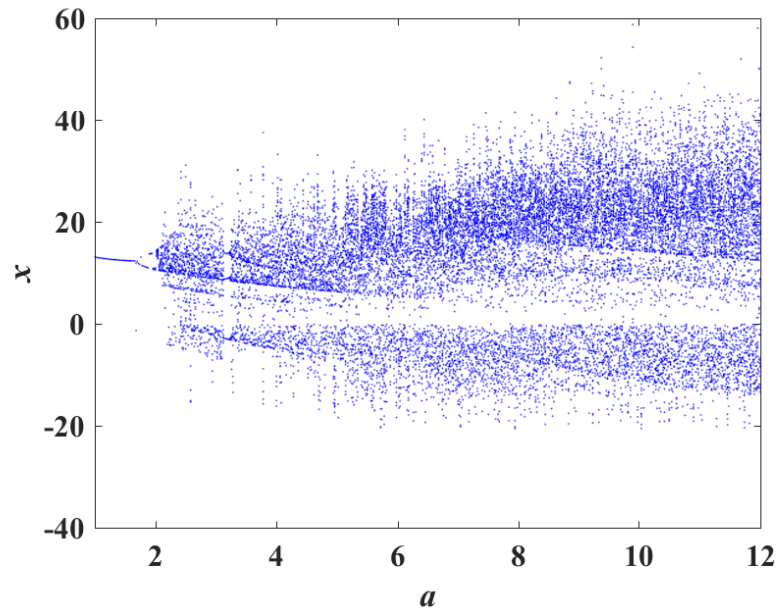

Fig. 11 Bifurcation diagram corresponding to $a$

is less than zero. Table 2 shows the different states of the 4D-HCS with different parameter $c$.

In addition, the bifurcation diagram in Fig. 14 shows that the system reversely bifurcates from the chaotic state to the periodic state when the parameter $c$ increases within $[-4,8]$, 


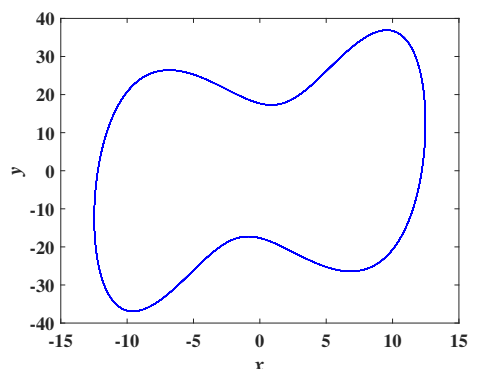

(a)

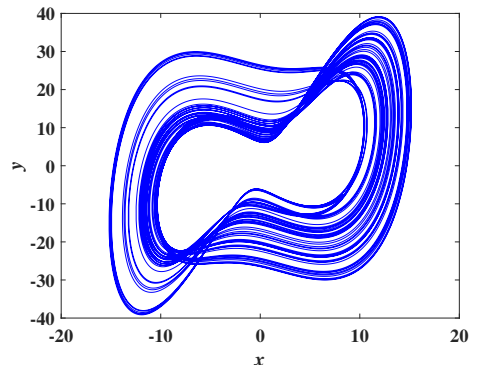

(b)

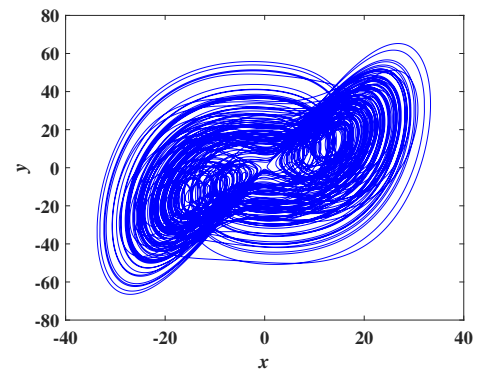

(c)

Fig. 12 Corresponding evolution process of the system with varying parameter $a$ : (a) Period with $a=1.4$, (b) Chaotic with $a=2.1$, (c) Hyper-chaotic with $a=8$

Table 2 Different system states corresponding to different values of $c$

\begin{tabular}{cccccl}
\hline Value of $c$ & $L E_{1}$ & $L E_{2}$ & $L E_{3}$ & $L E_{4}$ & State \\
\hline 7 & 0.0025 & -0.0034 & -0.9707 & -3.0283 & Period \\
4 & 3.0553 & -0.0079 & -0.0070 & -10.0403 & $\begin{array}{l}\text { Chaotic } \\
-2\end{array}$ \\
& 1.2517 & 0.3177 & -0.0281 & -14.4412 & $\begin{array}{l}\text { Hyper- } \\
\text { chaotic }\end{array}$ \\
\hline
\end{tabular}

which is consistent with its Lyapunov exponent spectrum in Fig. 13. Besides, Fig. 15 shows the phase diagram of the attractor on the $x-z$ plane when the parameter $c$ changes.

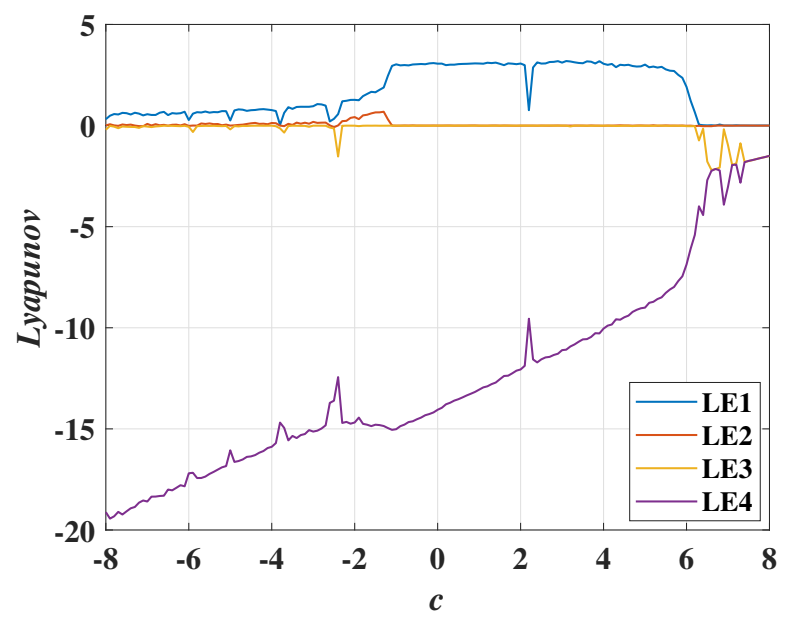

Fig. 13 Lyapunov exponent spectrum corresponding to $c$

\subsubsection{Dynamical map with parameters $a$ and $c$}

To further study the influence of parameters to the system state, we illustrate the dynamical map of the 4D-HCS. The dynamical map reflects the different dynamic characteristics of a system with the change of multiple parameters. The two-dimensional dynamic map of the 4D-HCS for parameters $a$ and $c$ is shown in Fig. 16, in which the green area

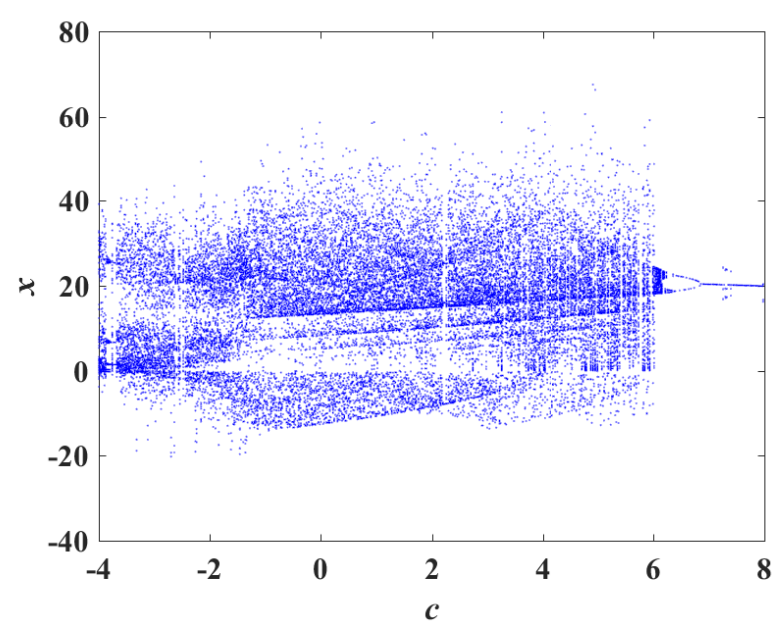

Fig. 14 Bifurcation diagram corresponding to $c$

marked with $P$ represents the periodic state, the blue area marked with $C$ represents the chaotic state, and the dark blue area marked with $H$ represents the hyper-chaotic state. It can be seen that when the parameter $c$ is less than -1 and $a$ changes within $[10,15]$, the 4D-HCS mainly shows hyperchaotic behaviors; when $c$ is greater than -1 and $a$ is set as a larger value, the system mainly shows chaotic behaviors; when $c$ is close to zero and $a$ is set as a smaller value, the system mainly show periodic behaviors.

4.2 Influence of memristor parameter on dynamic characteristics

The tri-valued memristor improves the dimension of the chaotic system and enlarges the number of parameters of the system. In this subsection, we analyze the influence of the memristor parameter to the dynamics of the 4D-HCS. 


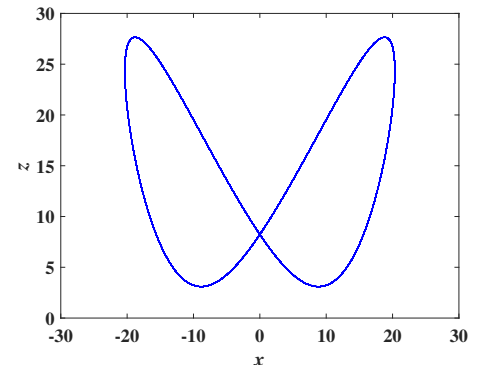

(a)

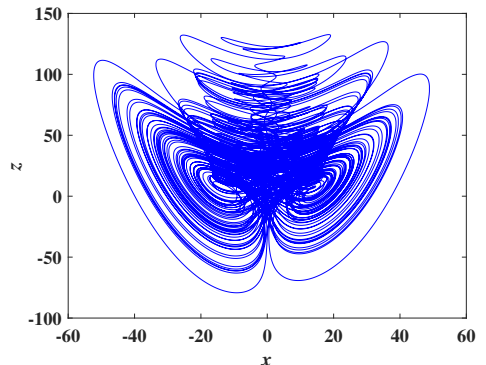

(b)

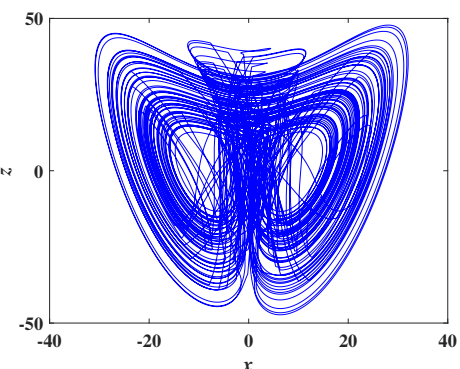

(c)

Fig. 15 Corresponding evolution process of the system with varying parameter $c$ : (a) Period with $c=7$, (b) Chaotic with $c=4$, (c) Hyper-chaotic with $c=-2$

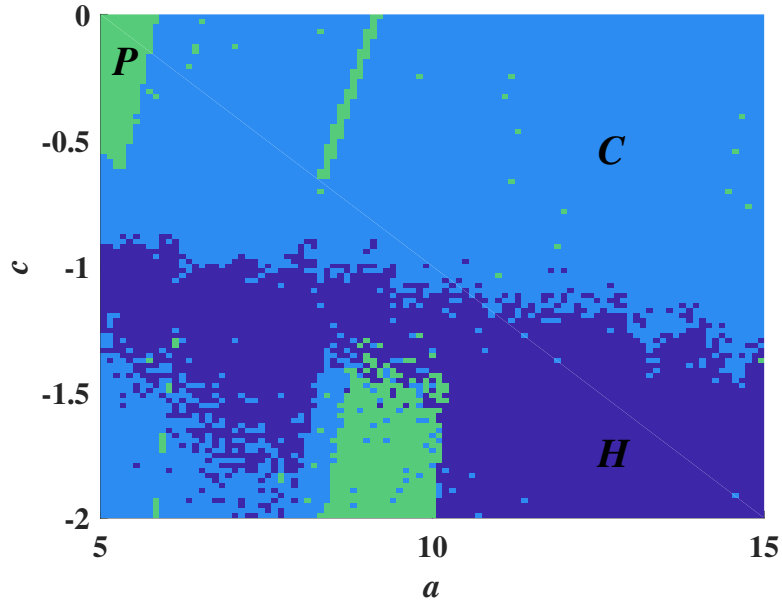

Fig. 16 Dynamical map of parameters $a$ and $c$

\subsubsection{Influence of the parameter $a_{0}$}

For the $q-\varphi$ relationship model of the memristor shown in Eq. (3), the memristor parameters are set as $\left[b_{0}, c_{0}, d_{0}, e_{0}\right]=[4$, $1,2.5,-1.5]$, and the system parameters are set as $[a, b, c$, $d]=[12,-1,-1.2,50]$. When the parameters $a_{0}$ varies within $[2.5,30]$, the new expression of $G(\varphi)$ can be obtained as follows:

$$
\begin{aligned}
G(\varphi) & =a_{0}+4 \operatorname{sgn}(\varphi+1)-2.5 \operatorname{sgn}(\varphi-1) \\
& = \begin{cases}a_{0}-4+2.5, & \varphi<-1 \\
a_{0}+4+2.5, & -1<\varphi<1 \\
a_{0}+4-2.5, & \varphi>1\end{cases}
\end{aligned}
$$

The Lyapunov exponent spectrum and bifurcation diagram of variable $x$ can be calculated with the change of parameter $a_{0}$ and they are shown in Fig. 17 and Fig. 18, respectively.

Fig. 17 shows that when $a_{0}$ increases within $[2.5,5]$, the system shows hyper-chaotic behaviors, and Fig. 18 shows that the system changes from the hyper-chaotic (and chaotic) state to the periodic state through inverse bifurcation with the increase of the parameter $a_{0}$.

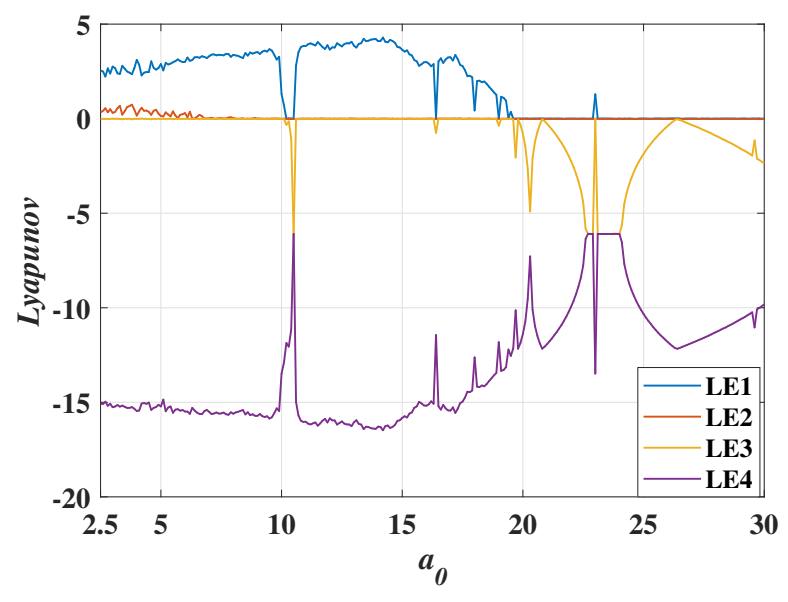

Fig. 17 Lyapunov exponent spectrum corresponding to $a_{0}$

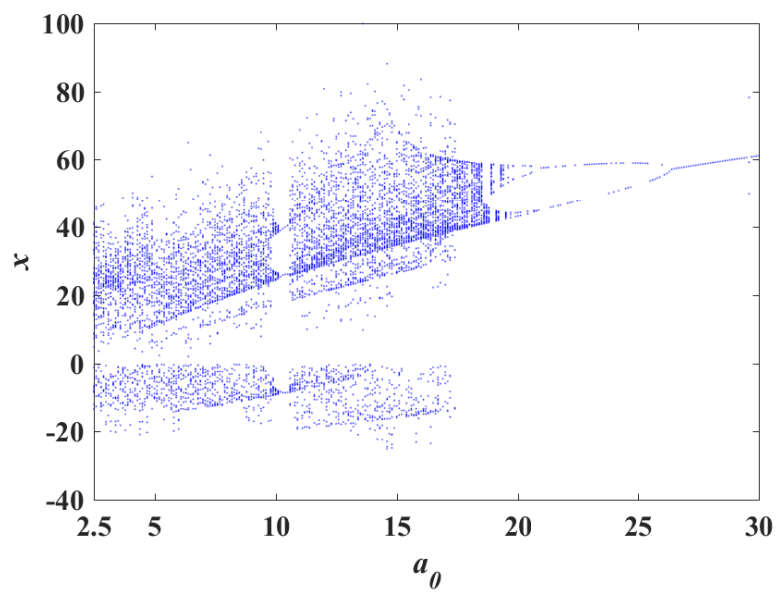

Fig. 18 Bifurcation diagram corresponding to $a_{0}$

Obviously, when the parameter $a_{0}$ varies within [2.5, $30]$, the memductance of the memristor changes makes the system state changes. This shows that the parameter space of the 4D-HCS is expanded, and its system state is affected by the memductance of memristor. The 4D-HCS still has no 
equilibrium point when $G(\varphi)$ is positive, and also shows the hidden oscillation phenomenon.

\subsubsection{Influence of the parameter $d_{0}$}

Similar to the analysis of parameter $a_{0}$, we set the parameter $\left[a_{0}, b_{0}, c_{0}, e_{0}\right]=[8,4,1,-1.5]$, and the parameter $d_{0}$ varies from $[-1,25]$. The new expression of $G(\varphi)$ is obtained as follows:

$$
\begin{aligned}
G(\varphi) & =8+4 \operatorname{sgn}(\varphi+1)-d_{0} \operatorname{sgn}(\varphi-1) \\
& = \begin{cases}8-4+d_{0}, & \varphi<-1 \\
8+4+d_{0}, & -1<\varphi<1 \\
8+4-d_{0}, & \varphi>1\end{cases}
\end{aligned}
$$

Keep other conditions unchanged and calculate the Lyapunov exponent spectrum and bifurcation diagram of variable $x$ with the change of parameter $d_{0}$ and show them in Fig. 19 and Fig. 20, respectively.

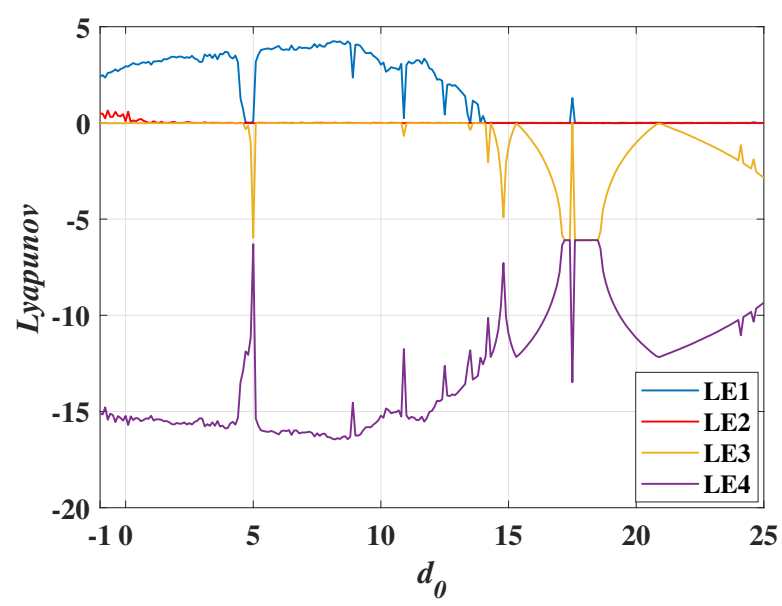

Fig. 19 Lyapunov exponent spectrum corresponding to $d_{0}$

It can be seen that with the change of parameter $d_{0}$, the 4D-HCS changes from the hyper-chaotic (and chaotic) state to the periodic state through the inverse bifurcation, which is similar to the evolution of the system with the change of the parameter $a_{0}$.

\subsubsection{Dynamical map with varying $a_{0}$ and $d_{0}$}

The dynamical map of the 4D-HCS for parameters $a_{0}$ and $d_{0}$ is shown in Fig. 21, in which the green area marked with $P$ represents the periodic state, the blue area marked with $C$ represents the chaotic state, and the dark blue area marked with $H$ represents the hyper-chaotic state. Fig. 21 shows the different dynamic characteristics of the system with the change of the parameters $a_{0}$ and $d_{0}$. Specifically, when the parameters $a_{0}$ and $d_{0}$ are both small values, the 4D-HCS

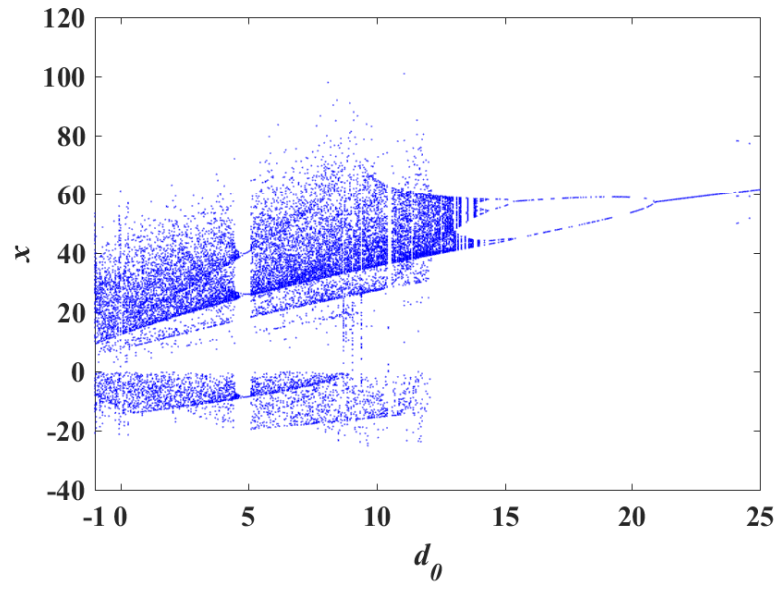

Fig. 20 Bifurcation diagram corresponding to $d_{0}$

mainly shows hyper-chaotic behaviors; when the $a_{0}$ and $d_{0}$ increase, the 4D-HCS mainly shows chaotic state. In addition, when the parameters $a_{0}$ and $d_{0}$ are set as appropriate values, the system shows periodic behaivors.

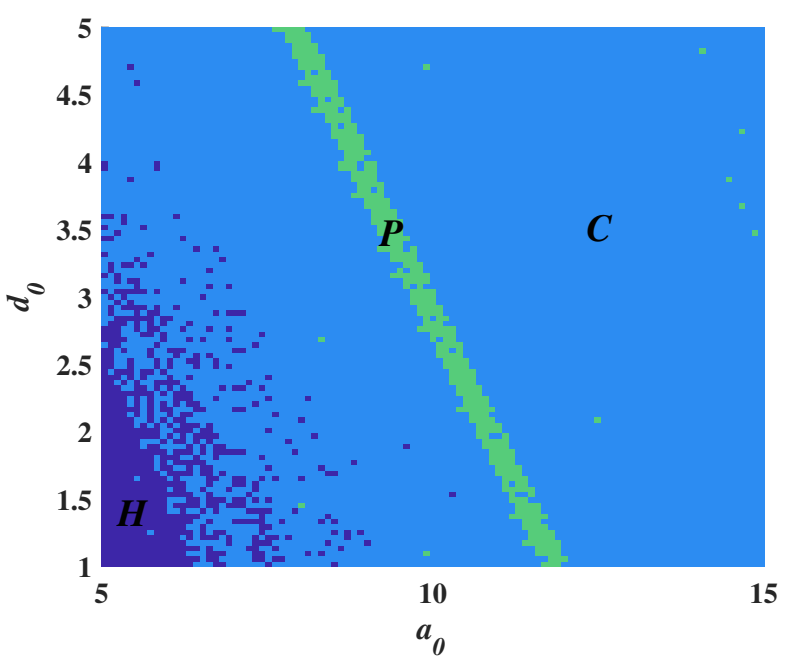

Fig. 21 Dynamical map of parameters $a_{0}$ and $d_{0}$

\subsection{Power spectrum analysis}

In this section, we analyze the power spectrum of the 4DHCS to further demonstrate the evolution of its state.

The power spectrum is obtained by performing the Fourier transform to the output sequence of the 4D-HCS, and it can be used to distinguish the difference of dynamic states. It is well-known that the power spectrum of a periodic signal is a discrete spectrum, and the power spectrum of an aperiodic signal is a continuous spectrum. For an aperiodic signal, its 
power spectrum is continuous and there are a large number of peaks in its corresponding power spectrum, this is mainly caused by a large number of period-doubling bifurcations in chaos. In addition, for a noise signal, its power spectrum is continuous and smooth.

Fig. 22 shows the phase diagram of different attractors in the $y-z$ plane and the corresponding power spectrum when set the system parameters as $a=12, b=-1, d=50$. Fig. 22(a) shows the period 1 attractor with $c=7$, and the corresponding power spectrum has a peak in Fig. 22(b). Fig. 22(c) shows the period 2 attractor with $c=6.35$, and the corresponding power spectrum has two peaks in Fig. 22(d). Obviously, the power spectrum of the above two periodic signals are all discrete spectrum. Fig. 22(e) shows the hyper-chaotic attractor with $c=-2$, and the corresponding power spectrum is shown in Fig. 22(f), which is a continuous spectrum, and a large number of peaks are formed due to period-doubling bifurcation.

Therefore, power spectrum analysis can effectively distinguish and compare the periodic signal, chaotic signal and noise signal of the system.

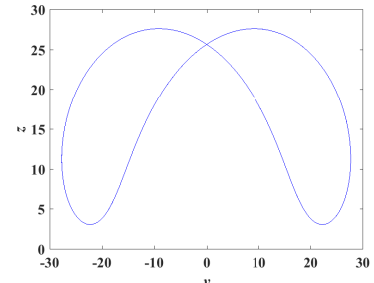

(a)

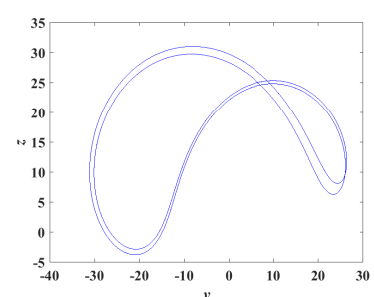

(c)

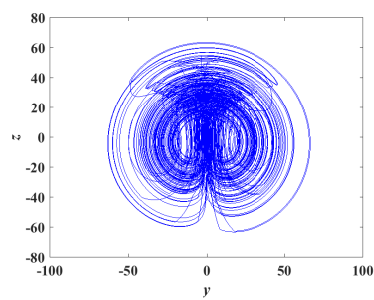

(e)

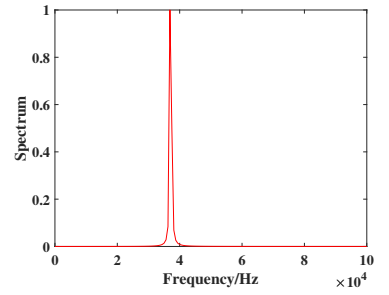

(b)

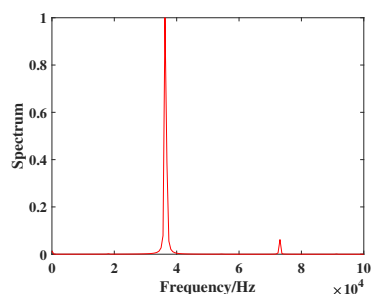

(d)

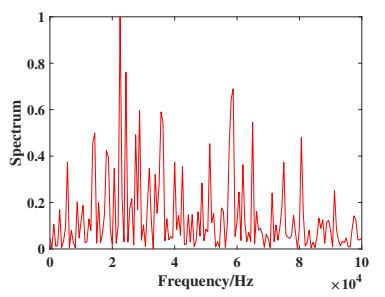

(f)
Fig. 22 Attractor and corresponding power spectrum: (a) and (b) period-1, (c) and (d) period-2, (e) and (f) hyper-chaotic

\subsection{Initial sensitivity}

A chaotic systems is also sensitive to the changes in its initial values. With slightly different initial values, the chaotic system can produce completely different sequences. The sensitivity of a chaotic system can be analyzed by measuring the correlation the two sequences generated by different initial values. The correlation coefficient which is defined by Eq. (13):

$C o=\frac{E\left[\left(X_{t}-\mu_{X}\right)\left(Y_{t}-\mu_{Y}\right)\right]}{\sigma_{X} \sigma_{Y}}$

where, $X_{t}$ and $Y_{t}$ are two sequences generated by the system under two slightly different initial values, $\mu$ and $\sigma$ represent the mean value and standard deviation of the sequence, $E[\cdot]$ is the expectation function [31]. A $C_{o}$ value closer 0 indicates the lower correlation between the two sequences, and further means the higher sensitivity of the chaotic system to its initial values.

For the 4D-HCS, we fix the system parameters $[a, b, c$, $d]=[12,-1,-2,50]$, and slightly change each variable in $\left[x_{0}\right.$, $\left.y_{0}, z_{0}, w_{0}\right]$ with a $10^{-8}$ difference. Taking the variable $x_{0}$ as an example and let $x_{0}{ }^{\prime}=x_{0}+10^{-8}$, then the system generates a different sequence pair $\left(X_{1}, X_{2}\right)$ under initial values $\left[x_{0}, 0.1\right.$, $0.1,0.1]$ and $\left[x_{0}{ }^{\prime}, 0.1,0.1,0.1\right]$. In the same way, slightly change the $y_{0}, z_{0}$ and $w_{0}$ to get $\left(Y_{1}, Y_{2}\right),\left(Z_{1}, Z_{2}\right)$ and $\left(W_{1}, W_{2}\right)$. Then calculate the correlation values of these sequence pairs and their results are shown in Table 3. It can be seen that the correlation values of all the sequence pairs are very close to 0 , which indicates that the 4D-HCS is extremely sensitive to changes of its initial values. To visually show the sensitivity, we plot these sequence pairs $\left(X_{1}, X_{2}\right),\left(Y_{1}, Y_{2}\right),\left(Z_{1}, Z_{2}\right)$ and $\left(W_{1}, W_{2}\right)$ in Fig. 23 . It clearly shows the difference between each sequence pair generated by the system with slightly different initial values. Therefore, the 4D-HCS is extremely sensitive to its initial values.

\subsection{Coexistent attractors analysis}

The 4D-HCS not only has hidden attractors, but also coexistent attractors. Fix the system parameters as $a=12, b=-1$ and $d=50$, and change the parameter $c$. When $c$ is set as an appropriate value, the 4D-HCS can obtain coexistent attractors under the symmetrical initial values $[0.1,0.1,0.1,0.1]$ and $[-0.1,-0.1,0.1,0.1]$. Different parameters $c$ can result in different states of the system.

Under symmetrical initial conditions, the phase diagrams of the coexistent period attractors and the coexistent chaotic attractors of the system are shown in Fig. 24 and Fig. 25, respectively.

In Fig. 24 and Fig. 25, the red area represents the trajectory with initial values $[-0.1,-0.1,0.1,0.1]$, while the blue 
Table 3 Correlation values of different sequences

\begin{tabular}{ccccc}
\hline \multirow{2}{*}{ Initial values } & \multicolumn{4}{c}{ Correlation coefficient } \\
\cline { 2 - 5 } & Correlation of $X_{1}, X_{2}$ & Correlation of $Y_{1}, Y_{2}$ & Correlation of $Z_{1}, Z_{2}$ & Correlation of $W_{1}, W_{2}$ \\
\hline$x_{0}-x_{0}^{\prime}$ case & -0.0643 & -0.0612 & -0.1663 & -0.0576 \\
$y_{0}-y_{0}^{\prime}$ case & -0.1252 & -0.0768 & 0.1275 & 0.0392 \\
$z_{0}-z_{0}^{\prime}$ case & 0.0912 & 0.0990 & -0.0543 & 0.0643 \\
$w_{0}-w_{0}^{\prime}$ case & 0.0355 & 0.0468 & 0.0075 & -0.0332 \\
\hline
\end{tabular}

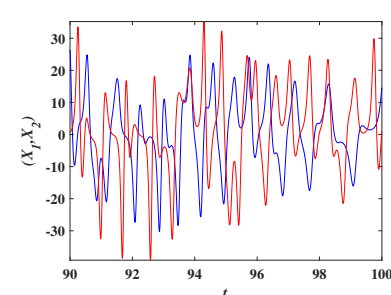

(a)

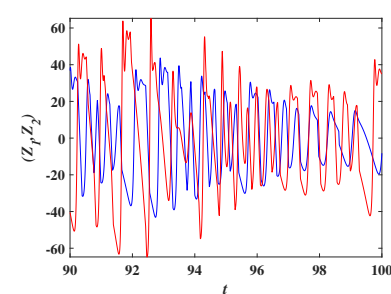

(c)

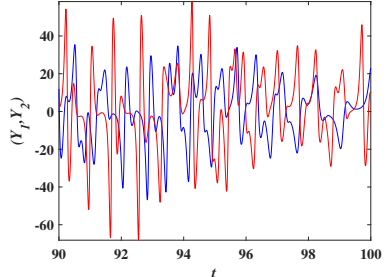

(b)

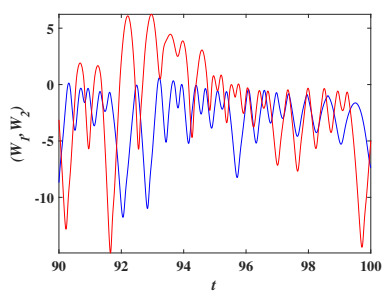

(d)
Fig. 23 The timing diagrams of the sequences under the case of $x_{0}-x_{0}{ }^{\prime}$ : (a) $\left(X_{1}, X_{2}\right)$, (b) $\left(Y_{1}, Y_{2}\right),(\mathbf{c})\left(Z_{1}, Z_{2}\right),(\mathbf{d})\left(W_{1}, W_{2}\right)$

Table 4 Each type of coexistent attractors

\begin{tabular}{|c|c|c|c|c|c|}
\hline \multirow{2}{*}{ Value of $c$} & \multicolumn{4}{|c|}{ Lyapunov exponent } & \multirow{2}{*}{ Type } \\
\hline & $L E_{1}$ & $L E_{2}$ & $L E_{3}$ & $L E_{4}$ & \\
\hline 6.4 & 0.0029 & -0.0046 & -0.1500 & -4.4483 & Period-1 \\
\hline 6.3 & 0.0054 & -0.0019 & -0.7340 & -3.9696 & Period-2 \\
\hline 6.25 & 0.0008 & -0.0036 & -0.1672 & -4.5799 & Period-4 \\
\hline 6.17 & 0.9388 & 0.0018 & -0.0052 & -5.7653 & Chaotic \\
\hline 2.4 & 3.1245 & -0.0054 & -0.0056 & -11.7135 & Chaotic \\
\hline-1.2 & 2.4566 & 0.3086 & -0.0064 & -14.9588 & $\begin{array}{l}\text { Hyper- } \\
\text { chaotic }\end{array}$ \\
\hline
\end{tabular}

area represents the trajectory with initial values $[0.1,0.1$, $0.1,0.1]$.

As a result, under the symmetrical initial values $[ \pm 0.1$, $\pm 0.1,0.1,0.1]$, a total of five types of coexistent attractors appear when the parameter $c$ changes, as shown in Table 4 .

\section{Comparison with the Binary-valued Memristor-based Chaotic System}

In this section, we compared the 4D-HCS with binary-valued memristor-based chaotic system to further illustrate the advantages of the tri-valued memristor.

\subsection{The binary-valued memristor model}

In [14], Itoh etal. proposed a binary-valued memristor model, whose $q-\varphi$ relationship is described by a symmetric piecewise linear function, and its general expression is shown in Eq. (14).

$q=\beta \varphi+0.5(\alpha-\beta)(|\varphi+\delta|-|\varphi-\delta|)$

where $\alpha, \beta$ and $\delta$ are positive parameters. The corresponding memductance $G^{\prime}$ can be described by Eq. (15).

$$
\begin{aligned}
G^{\prime}(\varphi) & =\beta+0.5(\alpha-\beta)[\operatorname{sgn}(\varphi+\delta)-\operatorname{sgn}(\varphi-\delta)] \\
& = \begin{cases}\alpha, & |\varphi|<\delta \\
\beta, & |\varphi|>\delta\end{cases}
\end{aligned}
$$

Similar to the tri-value memristor, setting $\alpha=9, \beta=4$, and $\delta=1$. Then the $G^{\prime}(\varphi)$ has two stable memductance $9 \mathrm{~S}$ and $4 \mathrm{~S}$. Applying a sinusoidal signal $v(t)=v_{0} \sin (\omega t)$ to the memristor, and taking $v_{0}=4, \omega=4$, and the initial value $\varphi(0)=-1.5$. The $v-i$ curve and $t-G^{\prime}$ curve can be obtained as shown in Fig. 26.

\subsection{Simulation and comparison}

Next, we introduce the $G^{\prime}(\omega)$ to replace the $G(\omega)$ in Eq. (8) to obtain a new system as shown in Eq. (16).

$$
\left\{\begin{array}{l}
\dot{x}=a(y-x) \\
\dot{y}=c y-x z \\
\dot{z}=x y-b z-d G^{\prime}(w) \\
\dot{w}=z
\end{array}\right.
$$

where, the $G^{\prime}(\omega) \neq 0$, which indicates that the system has no equilibrium point. 


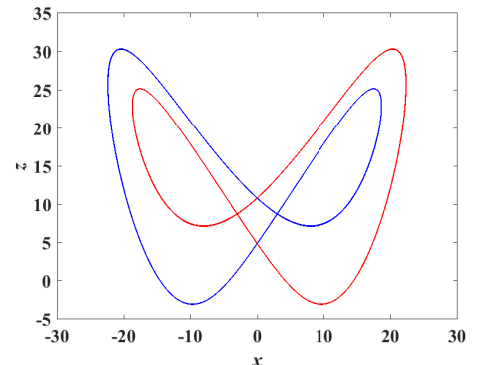

(a)

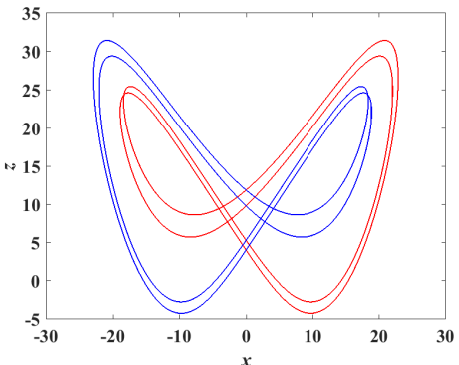

(b)

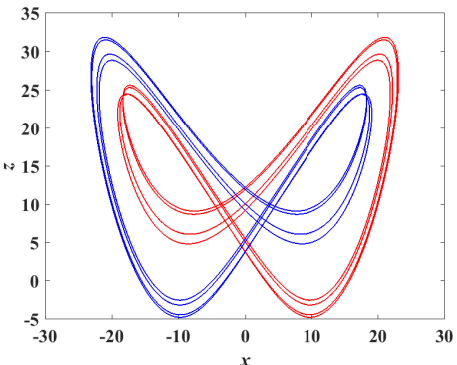

(c)

Fig. 24 Coexistent period attractors of the system on the $x-z$ plane: (a) Period-1 with $c=6.4$, (b) Period-2 with $c=6.3$, (c) Period-4 with $c=6.25$

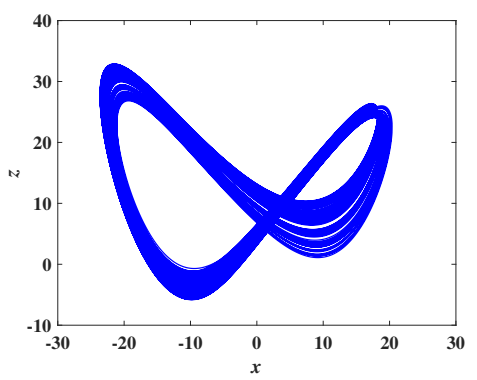

(a)

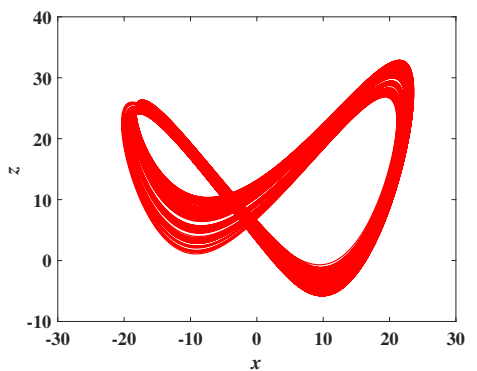

(d)

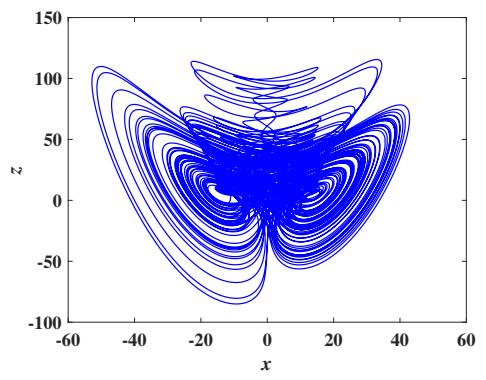

(b)

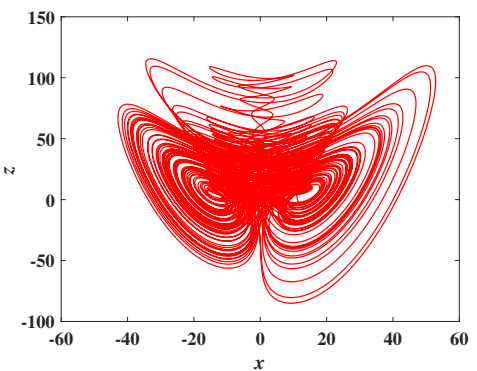

(e)

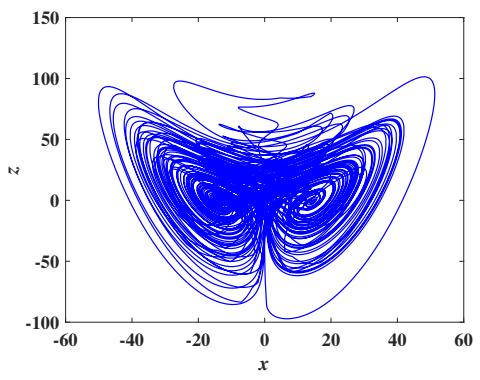

(c)

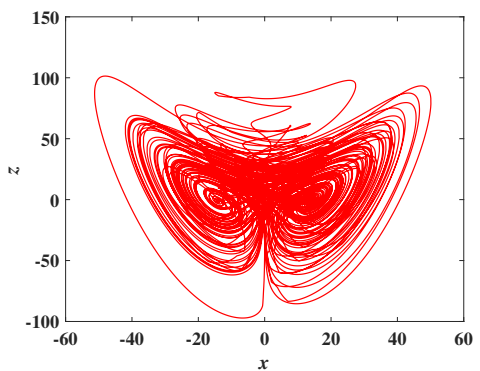

(f)

Fig. 25 Coexistent chaotic attractors of the system on the $x$ - $z$ plane: (a) and (d) Chaotic with $c=6.17$, (b) and (e) Chaotic with $c=2.4$, (c) and (f) Hyper-chaotic with $c=-1.2$

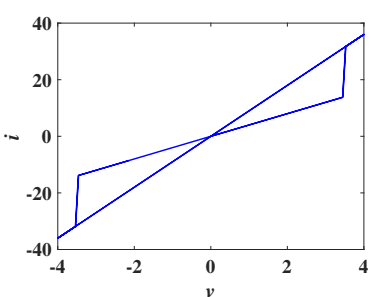

(a)

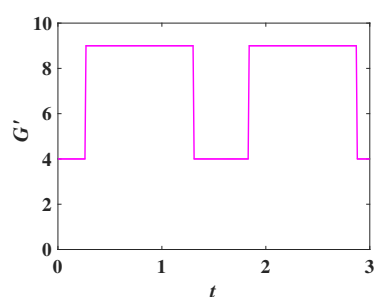

(b)
Fig. 26 The characteristic curve of the binary-valued memristor model: (a) $v$ - $i$ curve, (b) $t-G^{\prime}$ curve

Setting the system parameters as $a=12, b=-1, c=-1.2$ and $d=50$, and its initial values as $\left[x_{0}, y_{0}, z_{0}, w_{0}\right]=[0.1,0.1,0.1$, $0.1]$, the Lyapunov exponent are calculated as $L E_{1}=3.0002$,
$L E_{2}=0.0456, L E_{3}=-0.0234$ and $L E_{4}=-15.2225$, which indicates that the system shows chaotic behavior, and has hidden attractors as shown in Fig. 27.

Table 5 compares the simulation results of Eq. (8) and Eq. (16). It can be seen that under the same system parameters and initial conditions, both systems have hidden attractors. In addition, the system based on the tri-valued memristor has the unique characteristics of hyper-chaos and coexistence attractors, which indicates that the tri-valued memristor has a great advantage in enhance the chaos complexity.

\section{Hardware Implementation and Application}

In this section, we implement the hardware circuit platform of the 4D-HCS and apply it to design PRNG. 


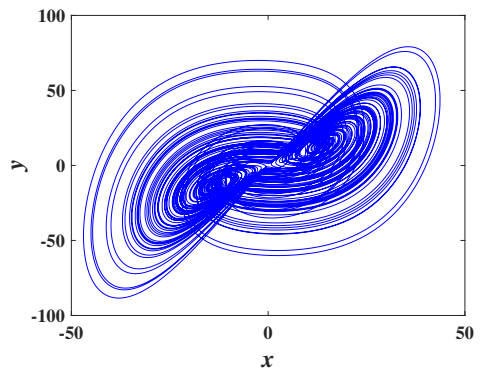

(a)

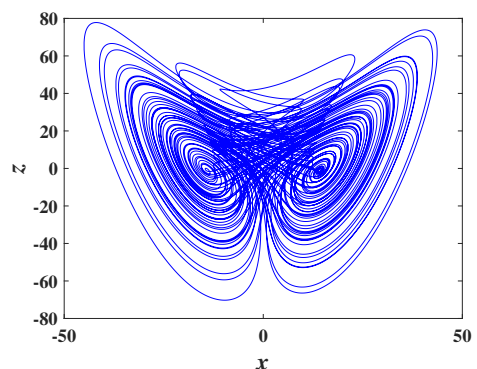

(b)

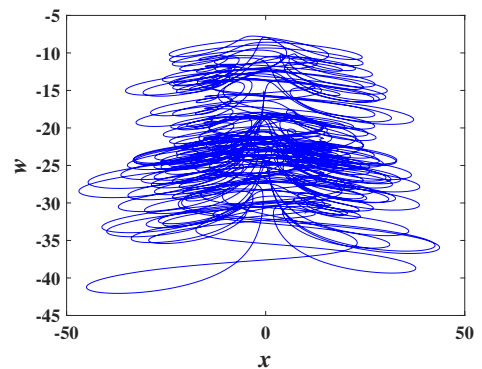

(c)

Fig. 27 Phase diagram of the chaotic attractors: (a) $x-y$, (b) $x-z$, (c) $x-w$

Table 5 The results of comparison

\begin{tabular}{ccccccccc}
\hline Memristor & System & $L E_{1}$ & $L E_{2}$ & $L E_{3}$ & $L E_{4}$ & Hidden attractors & Coexistent attractors & Hyper-chaotic \\
\hline Tri-valued & Eq. (8) & 2.4566 & 0.3086 & -0.0064 & -14.9588 & True & True \\
Binary-valued & Eq. (16) & 3.0002 & 0.0456 & -0.0234 & -15.2225 & True & False \\
\hline
\end{tabular}

\subsection{Hardware Implementation of the 4D-HCS}

To further verify the easy implementation of the 4D-HCS, we use an improved modular circuit design scheme to construct the hardware circuit of the system. In numerical simulation, the range of the system variables is much larger than the linear dynamic range $( \pm 13.5 \mathrm{~V})$ of the operational amplifier. Therefore, to avoid nonlinear distortion, the proportional compression transformation and time scale transformation must be performed first. For Eq. (8), let $k x \rightarrow x$, $k y \rightarrow y, k z \rightarrow z, k w \rightarrow w$ and $t=\tau_{0} t$, where $k=50$ and $\tau_{0}=100$. Determine the parameters $a=12, b=-1, c=-1.2$ and $d=50$, the final system can be described by the following equations:

$$
\left\{\begin{array}{l}
\frac{d x}{d t}=1200(y-x) \\
\frac{d y}{d t}=-120 y-5000 x z \\
\frac{d z}{d t}=5000 x y+100 z-100 G(50 w) \\
\frac{d w}{d t}=100 z
\end{array}\right.
$$

The design result of the modular hyper-chaotic circuit is shown in Fig. 28, and the state equations of the circuit can be described by:

$$
\left\{\begin{array}{l}
\frac{d x}{d t}=-\frac{1}{R_{1} C_{1}} x-\frac{1}{R_{2} C_{1}}(-y) \\
\frac{d y}{d t}=-\frac{1}{R_{4} C_{2}} y-\frac{1}{10 R_{3} C_{2}} x z \\
\frac{d z}{d t}=-\frac{1}{10 R_{6} C_{3}} x(-y)-{\frac{1}{R_{5} C_{3}}}_{3}(-z)-\frac{1}{R_{9} C_{3}} G(50 w) \\
\frac{d w}{d t}=-\frac{1}{R_{12} C_{4}}(-z)
\end{array}\right.
$$

According to Eqs. (17) and (18), the parameters of the circuit can be determined as: $C_{1}=C_{2}=C_{3}=C_{4}=10 \mathrm{nF}, R_{1}=R_{2}=$ $83.3 \mathrm{k} \Omega, R_{3}=R_{6}=2 \mathrm{k} \Omega, R_{4}=833 \mathrm{k} \Omega, R_{5}=R_{9}=R_{12}=1 \mathrm{M} \Omega, R_{7}=$ $R_{8}=R_{10}=R_{11}=10 \mathrm{k} \Omega$. In addition, the operational amplifiers $\operatorname{TL} 084 \mathrm{CN}\left(U_{1}-U_{6}\right)$ and analogue multipliers $\operatorname{AD633}\left(A_{1}-A_{2}\right)$ with $\pm 15 \mathrm{~V}$ power are used to construct the circuit.

Fig. 29 shows the physical hyper-chaotic circuit, and Fig. 30 shows the experimental results. Comparing the experimental results with the simulation results in Fig. 7, we can observe that the theoretical analysis is consistent with the physical experiment. This further proves the reliability of the 4D-HCS.

\subsection{Application in pseudorandom number generator}

To further investigate the complex dynamics behaviors of the 4D-HCS, we further designed a PRNG to analyze the performance of its hyper-chaotic sequences. First, set the parameters $a=12, b=-1, c=-1.2$ and $d=50$. For the initial values $x_{0}, y_{0}, z_{0}$ within $[0.1,1]$ along $w_{0}=0.1$, we take an interval 0.1 to obtain 1000 combinations of initial values. Then generate hyper-chaotic sequences using the Runge-Kutta methods. The time length is set as $135 \mathrm{~s}$, and the step size is set as 0.001 . Remove the first 10000 numbers, and finally get 1000 sets of different hyper-chaotic sequences $X_{i}, Y_{i}, Z_{i}, W_{i}$ $(i=1,2, \ldots, 1000)$, each of which has a length of 125000 .

Next, a PRNG is designed to generate pseudorandom numbers (PRNs) and it is defined as:

$\left\{\begin{array}{l}X_{p i}=\bmod \left(\operatorname{round}\left(X_{i} \cdot 10^{4}\right), 256\right) \\ Y_{p i}=\bmod \left(\operatorname{round}\left(Y_{i} \cdot 10^{4}\right), 256\right) \\ Z_{p i}=\bmod \left(\operatorname{round}\left(Z_{i} \cdot 10^{4}\right), 256\right) \\ W_{p i}=\bmod \left(\operatorname{round}\left(W_{i} \cdot 10^{4}\right), 256\right)\end{array}\right.$ 

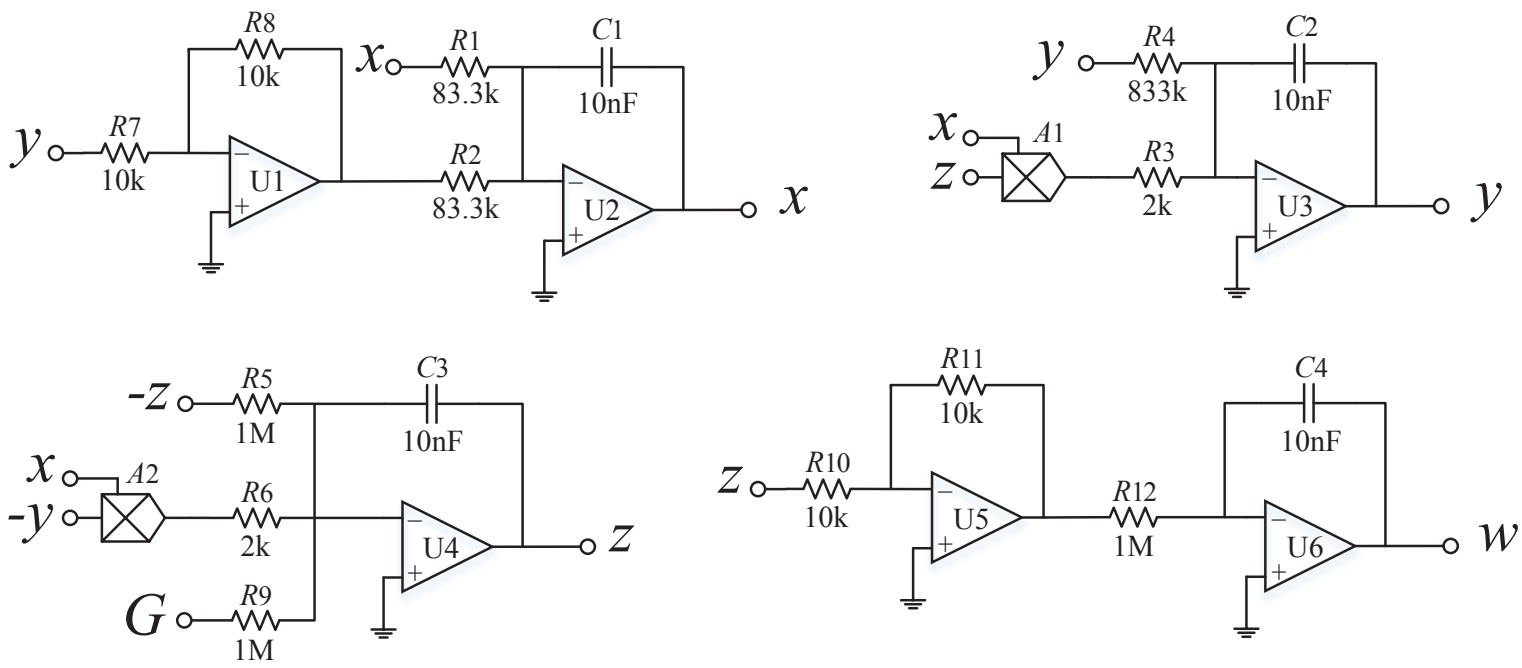

Fig. 28 Hyper-chaotic circuit diagram

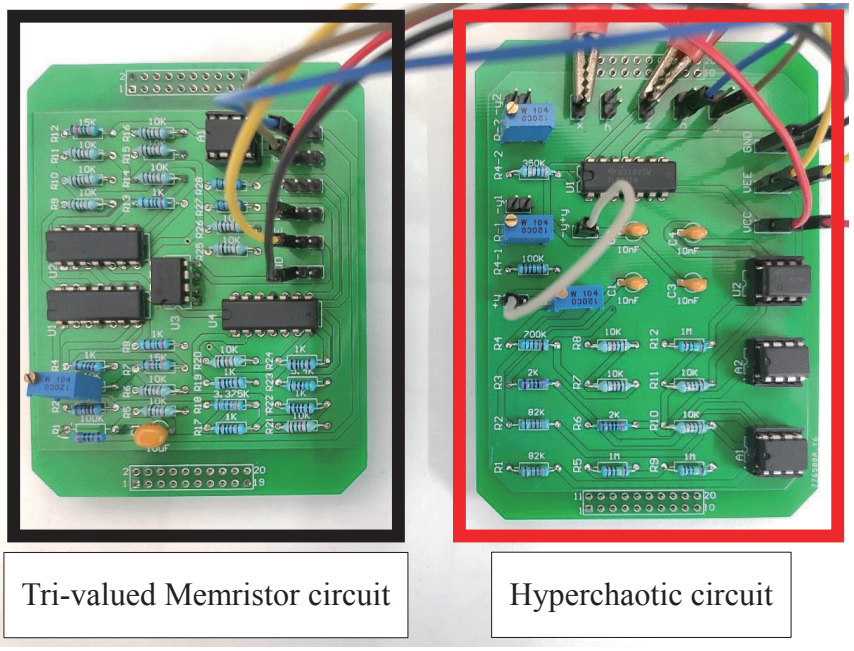

Fig. 29 Physical hyper-chaotic circuit

where round $(\cdot)$ is rounding operation, $\bmod (\cdot, 256)$ represents the remainder operation of 256, and the PRN sequences $\left\{X_{p i}\right.$, $\left.Y_{p i}, Z_{p i}, W_{p i}\right\}$ with a value range of 0 to 255 are obtained. Each PRN is represented by an 8-bit stream. Therefore, a pseudorandom binary stream of length $10^{6}$ bits can be obtained from each hyper-chaotic sequence.

A PRNG is expected to generate PRNs with high randomness. Here, we use the National Institute of Standards and Technology (NIST) SP800-22 test suit in [32] to test the performance of the pseudorandom bitstreams generated by the proposed PRGN. The NIST SP800-22 test includes 15 subtests. For each subtest, a $P$-value is generated and the sequence is considered to pass the subtest if the P-value is greater than the significance level $\alpha$, which is usually set as 0.01 .

Here, we choose the hyper-chaotic sequence sets $X$ and $Z$ as two examples to generate two binary stream sets with a sample size of 1000 and a sample length of $10^{6}$, which meet the test conditions described in [32]. The test results are given in Table 6 , where $P$-value $T$ represents the uniform distribution of $P$-values, which is calculated as:

$P-$ value $_{T}=\operatorname{igamc}\left(9 / 2, \chi^{2} / 2\right)$

where igamc $(\cdot)$ is the incomplete gamma function and:

$\chi^{2}=\sum_{i=1}^{10} \frac{\left(F_{i}-s / 10\right)^{2}}{s / 10}$

where the range of $P$-value is evenly divided into 10 subintervals, $F_{i}$ represents the number of $P$-value in the $i$ th subinterval, and $s$ represents the sample size. If the obtained $P$ value $_{T} \geq 0.0001$, the sequences can pass the corresponding subtest. And the minimum pass proportions are approximately 0.980 for a sample size 1000 .

The test results in Table 6 show that all $P$-value $T$ are larger than 0.0001 and all pass proportion are larger than or equal to the minimum pass rate of 0.980 , indicating that the obtained PRNs can pass all the 15 subtests. This indicates that the proposed PRNG using the 4D-HCS can generate a large number of PRNs with high randomness. Therefore, the 4D-HCS system has potential application in the field of cryptography.

\section{Conclusion}

This paper proposes a new 4D-HCS based on the tri-valued memristor. First, the mathematical model of memristor is analyzed and its equivalent circuit is verified. Then, the 4DHCS is constructed by introducing the memristor into an existing chaotic system. Stability analysis results show that the 


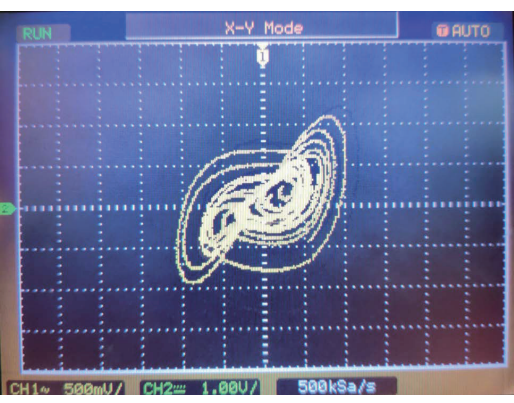

(a)

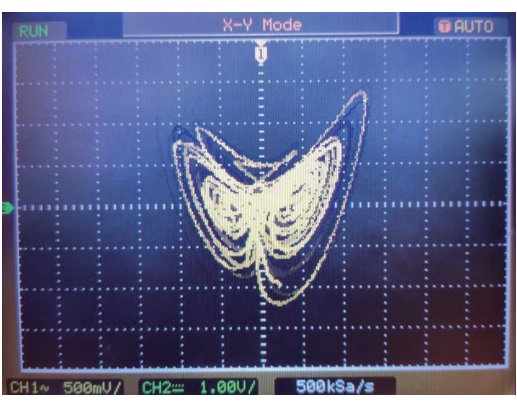

(b)

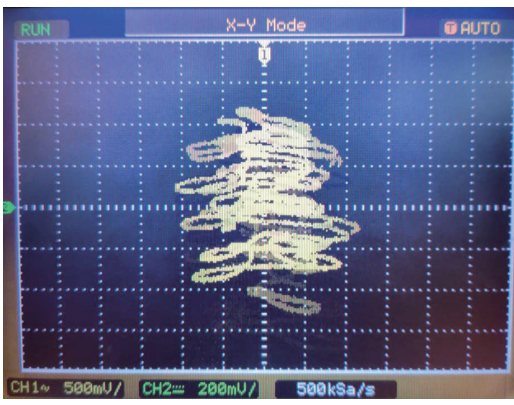

(c)

Fig. 30 Experimental results: (a) $x-y$ plane, (b) $x-z$ plane, (c) $x-w$ plane

Table 6 NIST test results of linear congruence and hyperchaotic pseudorandom sequences

\begin{tabular}{|c|c|c|c|c|c|}
\hline \multirow[b]{2}{*}{ No. } & \multirow[b]{2}{*}{ Test items } & \multicolumn{2}{|c|}{ PRNs obtained by $X$} & \multicolumn{2}{|c|}{ PRNs obtained by $Z$} \\
\hline & & $P$-value $T$ & Proportion & $P$-value $T$ & Proportion \\
\hline 01 & Frequency & 0.350485 & 0.987 & 0.767582 & 0.990 \\
\hline 02 & Block Frequency & 0.071620 & 0.993 & 0.317565 & 0.991 \\
\hline \multirow{2}{*}{03} & Cumulative Sums (F) & 0.488534 & 0.987 & 0.701366 & 0.992 \\
\hline & Cumulative Sums (R) & 0.705466 & 0.989 & 0.380407 & 0.989 \\
\hline 04 & Runs & 0.861264 & 0.989 & 0.154629 & 0.987 \\
\hline 05 & Longest Run & 0.296834 & 0.990 & 0.249284 & 0.996 \\
\hline 06 & Rank & 0.572847 & 0.984 & 0.536163 & 0.987 \\
\hline 07 & FFT & 0.522100 & 0.989 & 0.317565 & 0.987 \\
\hline 08 & Non-Overlapping Template * & 0.119508 & 0.980 & 0.170922 & 0.983 \\
\hline 09 & Overlapping Template & 0.419021 & 0.992 & 0.145326 & 0.994 \\
\hline 10 & Universal & 0.496351 & 0.988 & 0.124476 & 0.981 \\
\hline 11 & Approximate Entropy & 0.454053 & 0.997 & 0.887645 & 0.986 \\
\hline 12 & Random Excursions * & 0.294973 & 0.982 & 0.218048 & 0.988 \\
\hline 13 & Random Excursions Variant * & 0.472198 & 0.981 & 0.385257 & 0.983 \\
\hline \multirow{2}{*}{14} & Serial (1st sub-test) & 0.260930 & 0.994 & 0.313041 & 0.991 \\
\hline & Serial (2st sub-test) & 0.880145 & 0.993 & 0.585209 & 0.992 \\
\hline 15 & Linear Complexity & 0.322135 & 0.996 & 0.377007 & 0.987 \\
\hline
\end{tabular}

* Non-overlapping template test, Random excursions test, and Random excursions variant test are comprised of 148, 8, and 18 subtests, respectively. The result with the lowest pass proportion of multiple subtests is reported.

4D-HCS has no equilibrium point and thus has hidden attractors. The dynamic characteristics of the 4D-HCS are analyzed in aspects of the Poincaré mapping, bifurcation diagram, Lyapunov exponential spectrum and power spectrum. The analysis results verify that the introduction of tri-valued memristor can greatly enhance the chaos complexity of existing chaotic system. Besides, the characteristics of the coexistent attractors of the 4D-HCS are analyzed, and the results proves that the 4D-HCS can show five types of coexistent attractors. Furthermore, we compared the simulation results of the binary-valued memristor-based chaotic system. The results show that the introduction of the tri-valued memristor is beneficial to the system to produce more complex characteristics. To show the easy implementation of the 4D-HCS, we build a physical circuit to implement the system and the implementation results show the correctness of the theoretical analysis. Finally, a PRNG is designed using the 4D-HCS. Test results shows that the PRNG can generate random numbers with high randomness. Therefore, the application of such kind of memristive hyper-chaotic systems in cryptography deserve to further explore in the future.

Acknowledgements This work was supported by the National Natural Science Foundation of China (Grant No. 61871429), and the Natural Science Foundation of Zhejiang Province (Grant No. LY18F010012),

\section{Declarations}

\section{Data Availability Statement}

The datasets generated during and/or analyzed during the current study are available from the corresponding author on reasonable request. 


\section{Conflict of interest}

The authors declare that they have no conflict of interest.

\section{Compliance with ethical standards}

\section{References}

1. Lorenz, E, N. Deterministic Nonperiodic Flow, Journal of the Atmospheric Sciences, 20, 130-141 (1963)

2. Fan, C., Ding, Q. Analysis and resistance of dynamic degradation of digital chaos via functional graphs. Nonlinear Dyn, 103, 10811097 (2021)

3. Wan, Y., Cao, J., Wen, G. Quantized Synchronization of Chaotic Neural Networks With Scheduled Output Feedback Control. IEEE Transactions on Neural Networks and Learning Systems, 28, 2638-2647 (2017)

4. Zhou, L., Tan, F. A chaotic secure communication scheme based on synchronization of double-layered and multiple complex networks. Nonlinear Dyn 96, 869-883 (2019)

5. Li, RG., Wu, HN. Secure communication on fractionalorder chaotic systems via adaptive sliding mode control with teaching-learning-feedback-based optimization. Nonlinear Dyn, 95, 1221-1243 (2019)

6. Hua, Z., Zhang, Y., Zhou, Y. Two-Dimensional Modular Chaotification System for Improving Chaos Complexity. IEEE Transactions on Signal Processing, 68, 1937-1949 (2020)

7. Hua, Z., Zhou, Y., Huang H. Cosine-transform-based chaotic system for image encryption. Information Sciences, 480, 403-419 (2019)

8. Wen, W., Wei, K., Zhang, Y., Fang, Y., Li, M. Colour light field image encryption based on DNA sequences and chaotic systems. Nonlinear Dyn, 99, 1587-1600 (2020)

9. Wang, X., Gao, M., Min, X., Lin, Z., Iu, H, H-C. On the Use of Memristive Hyperchaotic System to Design Color Image Encryption Scheme. IEEE Access, 8, 182240-182248 (2020)

10. Hua, Z., Zhu, Z., Yi, S., Zhang, Z., Huang, H. Cross-plane colour image encryption using a two-dimensional logistic tent modular map. Information Sciences, 546, 1063-1083 (2021)

11. Hua, Z., Li, J., Chen, Y., Yi, S. Design and application of an S-box using complete Latin square. Nonlinear Dyn, 104, 807-825 (2021)

12. Wang, X., Jin, C., Min, X., Yu, D., Iu, H, HC. An exponential chaotic oscillator design and its dynamic analysis. IEEE/CAA Journal of Automatica Sinica, 7, 1081-1086 (2020)

13. Xie, W., Wang, C., Lin, H. A fractional-order multistable locally active memristor and its chaotic system with transient transition, state jump. Nonlinear Dyn, 104, 4523-4541 (2021)

14. Itoh, M., Chua, L. Memristor oscillators. International Journal of Bifurcation and Chaos, 18, 3183-3206 (2008)

15. Wang, X., Fitch, A, L., Iu, H, HC., Sreeram, V., Qi, W. Implementation of an analogue model of a memristor based on a lightdependent resistor. Chinese Physics B, 21, 500-507 (2012)

16. Wang, X., Wang, G., Wang, X. Dynamic character analysis of a LDR, memristor-based chaotic system. Journal of Circuits, Systems and Computers, 23, 1450085.1-1450085.11 (2014)

17. Yu, F., Liu, L., Shen, H., Zhang, Z., Wan, Q. Multistability Analysis, Coexisting Multiple Attractors, and FPGA Implementation of $\mathrm{Yu}-$ Wang Four-Wing Chaotic System . Mathematical Problems in Engineering, 2020, 1-16 (2020)

18. Li, C., T, W, JC., Iu, H, HC., Lu, T. A Memristive Chaotic Oscillator With Increasing Amplitude and Frequency. IEEE Access, 6, 12945-12950 (2018)

19. Ye, X., Mou, J., Luo, C., Wang, Z. Dynamics analysis of Wienbridge hyperchaotic memristive circuit system. Nonlinear Dyn, 92, 923-933 (2018)
20. Tan, Q., Zeng, Y., Li, Z. A simple inductor-free memristive circuit with three line equilibria. Nonlinear Dyn, 94, 1585-1602 (2018)

21. Wang, X., Yu, J., Jin, C., Iu, H, HC., Yu, S. Chaotic oscillator based on memcapacitor and meminductor. Nonlinear Dyn, 96, 161-173 (2019)

22. Min, X., Wang, X., Zhou, P., Yu, S., Iu, H, HC. An Optimized Memristor-Based Hyperchaotic System With Controlled Hidden Attractors. IEEE Access, 7, 124641-124646 (2019)

23. Wang, X., Min, X., Zhou, P., Yu, D. Hyperchaotic Circuit Based on Memristor Feedback with Multistability and Symmetries. Complexity, 2020, 1-10 (2020)

24. Hua, Z., Zhou Y., Bao, B. Two-Dimensional Sine Chaotification System With Hardware Implementation. IEEE Transactions on Industrial Informatics, 16, 887-897 (2020)

25. Chua, L. Everything you wish to know about memristors but are afraid to ask. Radioengineering, 24, 319-368 (2015)

26. Wang, X., Zhang, X., Gao, M. A Novel Voltage-Controlled TriValued Memristor and Its Application in Chaotic System. Complexity, 2020, 1-8 (2020)

27. Biolek, D., Biolkova, V., Kolka, Z. Memristor pinched hysteresis loops: Touching points, Part I. 2014 International Conference on Applied Electronics, 37-40 (2014)

28. Hamed, E, M., Fouda, M, E., Radwan, A, G. Multiple Pinch-Off Points in Memristive Equations: Analysis and Experiments. IEEE Transactions on Circuits and Systems I: Regular Papers, 66, 30523063 (2019)

29. Lü, J., Chen, G. A new chaotic attractor coined. International Journal of Bifurcation and Chaos, 12, 659-661 (2002)

30. Chen, G., Ueta T. Yet another chaotic attractor. International Journal of Bifurcation and Chaos, 9, 1465-1466 (1999)

31. Hua, Z., Zhou, Y. Dynamic Parameter-Control Chaotic System. IEEE Transactions on Cybernetics, 46, 3330-3341 (2016)

32. Bassham, L., Rukhin, A., Soto, J., Nechvatal, J., Smid, M., Leigh, S., Levenson, M., Vangel, M., Heckert, N., Banks, D. A Statistical Test Suite for Random and Pseudorandom Number Generators for Cryptographic Applications, Special Publication (NIST SP). National Institute of Standards and Technology, Gaithersburg, MD (2010) 